Rhode Island College

Digital Commons @ RIC

$5-5-2020$

\title{
Preoperative Multimodal Analgesia in Total Hip Arthroplasty: A Retrospective Chart Review
}

Bonnie A. Blaney

Follow this and additional works at: https://digitalcommons.ric.edu/etd

Part of the Nursing Commons

\section{Recommended Citation}

Blaney, Bonnie A., "Preoperative Multimodal Analgesia in Total Hip Arthroplasty: A Retrospective Chart Review" (2020). Master's Theses, Dissertations, Graduate Research and Major Papers Overview. 346. https://digitalcommons.ric.edu/etd/346

This Major Paper is brought to you for free and open access by the Master's Theses, Dissertations, Graduate Research and Major Papers at Digital Commons @ RIC. It has been accepted for inclusion in Master's Theses, Dissertations, Graduate Research and Major Papers Overview by an authorized administrator of Digital Commons @ RIC. For more information, please contact digitalcommons@ric.edu. 


\title{
PREOPERATIVE MULTIMODAL ANALGESIA
}

\section{IN TOTAL HIP ARTHROPLASTY: A RETROSPECTIVE CHART REVIEW}

\author{
by
}

Bonnie A. Blaney RN BSN

A Major Paper Submitted in Partial Fulfillment

of the Requirements for the Degree of

Master of Science in Nursing

in

The School of Nursing

Rhode Island College

2020 


\begin{abstract}
The U.S. Department of Health and Human Services Pain Management Best Practices Interagency Task Force recommends procedure-specific, multimodal regimens and therapies in the perioperative period. Historically, joint arthroplasty has been known to require significant amounts of postoperative opioids to reduce pain and enable early mobilization; however, side effects such as sedation, nausea, and vomiting can delay early mobilization. Many accredited joint centers have adopted Enhanced Recovery After Surgery programs (ERAS) to provide optimal analgesic effect with decreased length of stay and narcotic consumption using a multimodal analgesic approach encompassing opioid and nonopioid analgesia. Despite the promotion of ERAS, this practice is not in place for hip arthroplasty at the clinical site, where treatment option is dependent on each practitioner preference. The purpose of this retrospective chart review was to determine whether there is a difference in postoperative opioid requirements and pain levels in the first 24 inpatient hours in adults over the age of 60 undergoing primary hip arthroplasty depending on the type of multimodal perioperative analgesia received. A sample of 200 charts meeting the study criteria were reviewed and study variables collected and analyzed using Excel. Results showed that $32 \%(n=64)$ of the 200 patients received preoperative analgesia. Of those, $89 \%(n=59)$ received a preoperative dose of acetaminophen. Compared to patients who did not receive preoperative analgesia, those who had two multimodal analgesia had a lower dose average of opioid consumption and pain scores within the first 24 hours postoperatively. The results underscore the benefits of preoperative multimodal use. Collaboratively, APRN could work with surgeons to implement ERAS, provide staff education, and evaluate pathway effectiveness.
\end{abstract}




\section{Acknowledgements}

I would like to thank my husband. It was with his urging that I started this journey. Without his constant support and encouragement, I would not have made it through. Thank you for taking on the role of two parents. You listened to me laugh, cry, and stress out over the littlest things; always telling me I could do it. You were my rock.

Matthew, my son, I thank you for all your understanding of the time taken away from you to complete my journey. The many hugs you gave me when I was stressed and nervous gave me the strength to push through whatever obstacle there was. I hope seeing how hard work pays off, will encourage you to seek out your dreams knowing that anything is possible.

For all my fellow classmates and professors, we have had an amazing journey. This path that we have made through these uncertain times is nothing short of amazing. It is from our friendships and support that we managed to find our way through the global pandemic and reach the finish line.

Finally, I dedicate this to my mom. Forever my cheerleader. I know that even though you could not see me finish my journey you always remained at my side. You had so much faith in me that I could complete this journey without you here. I made it mom, through all the obstacles life sent our way. I love you and thank you for always being my cheerleader. 


\section{Table of Contents}

Background/Statement of the Problem .................................................................. 1

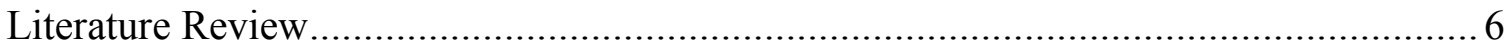

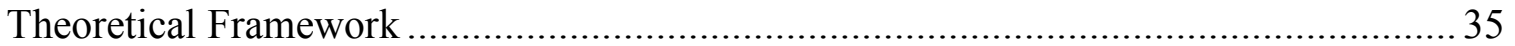

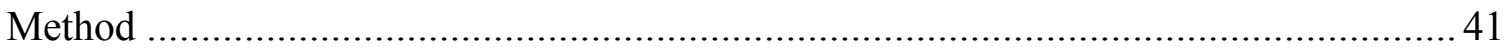

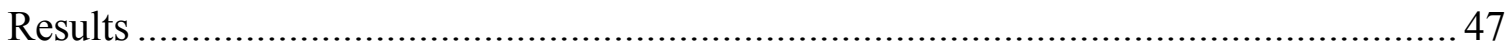

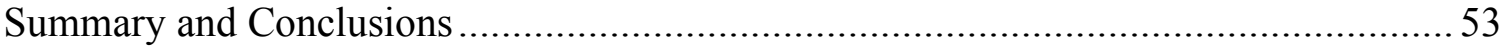

Recommendations and Implications for Advanced Nursing Practice ...........................56

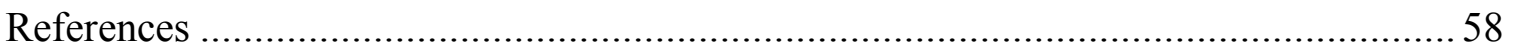

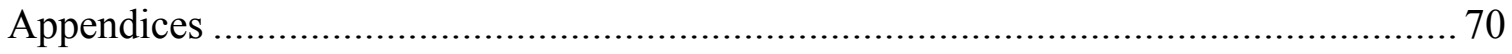


Preoperative Multimodal Analgesia in Total Hip Arthroplasty

\section{Background/Statement of the Problem}

Pain is defined as physical suffering caused from illness or injury (Pain, 2019). It is a sensation people will experience in their lifetime. What degree of pain experienced is based on each person's perception of pain. During the 1990's, those in healthcare began to change their perspective on pain in response to the American Pain Society recommendation that pain be assessed with the same intensity as other vital signs (Koepke et al., 2018). The Veterans Affairs Hospital Systems reacted by requiring pain be documented as the fifth vital sign, along with blood pressure, heart rate, respiration, and temperature measurements (Koepke et al., 2018). The Joint Commission on Accreditation of Healthcare Organizations (JCAHO) responded by educating health care professionals on the how to perform pain assessments and appropriate documentation of pain. In 2001, JCAHO required the documentation of pain on every patient. The requirement of documentation and treatment of pain became an integral part of a patient's treatment plan. With the change in clinical practice, there emerged a dramatic reciprocal increase in opioid prescriptions and overdoses (Hsu et al., 2019). In the year 1999, prior to practice changes, a total of 6,009 narcotic overdoses were recorded nationally (Centers for Disease Control and Prevention Web-based Injury Statistics Query and Reporting System [CDC WISQARS], 2019a). Most recent national statistics demonstrate a dramatic increase, with a total of 29,288 deaths from narcotic overdose in 2017 (CDC WISQARS, 2019b). This indicates an almost five-fold increase within 18 years.

In the 1990s there was an increase in opioid prescriptions with a reciprocal increase in opioid overdoses from natural and semi-synthetic opioids and methadone. 
This increase in opioid overdose deaths continued to rise until 2016, in which the death rate plateaued. In 2013 there was an increase in overdose deaths from synthetic opioids, those that were prescribed or illicitly manufactured, such as Tramadol and Fentanyl (CDC, 2019). The U.S. Department of Health and Human Services (HHS) in 2017 declared the opioid overdose deaths to be a public health emergency (HHS, 2017). The term opioid crisis or opioid epidemic was used to describe this emergency. The two terms are used interchangeably in literature and refer to the rapid rise in the administration of prescription and nonprescription opioid drugs (Koepke et al., 2018). This crisis is unique to the United States and is linked to the recommendation of frequent pain evaluations, pain documentation, and treatment in the 1990s.

Despite the ongoing increase in opioid overdoses, there still remains the challenge of managing pain without contributing to the opioid crisis. In an effort to control pain effectively, but limit opioid usage, the use of multimodal pain management modals was developed. Multimodal analgesia or multimodal pain management is referred to as the use of two or more pharmacologic analgesics from different pharmacologic classes. The medications target different pain pathways; the central nervous system and the peripheral nervous system to achieve optimal pain relief. The goals of multimodal analgesia are to improve the quality of analgesic effects, attain a more balanced analgesia, and decrease or minimize the adverse effects of analgesia while minimizing opioid use (Galloway et al., 2011).

The use of multimodal analgesia for postoperative patients was first extensively written about in the early 1990s by Kehlet and Dahl (1993). These authors explored the current use of unimodal treatment, and the ineffectiveness of achieving optimal pain 
relief without significant adverse effects. They theorized that since combination or multimodal therapy (the use two or more pharmacologic analgesics from different pharmacologic classes) was shown to be effective in less complex, small surgeries, it should be effective for larger more complex cases. The authors recommended multimodal or "balanced" anesthesia which would provide pain relief with a reduction in opioids and a subsequent decrease in undesirable side effects. The authors recommended further research to identify potentially effective combinations (Kehlet \& Dahl, 1993).

The use of multimodal analgesia has been recommended by the U.S. Department of Health and Human Services Pain Management Best Practices Interagency Task Force. The task force recommended "procedure-specific, multimodal regimens and therapies" (U.S Department of Health \& Human Services, 2018, p.11) in the perioperative period. These include nonopioid analgesic medications in addition to nerve blocks. The task force found that despite these recommendations, these services are underutilized in the perioperative setting. In a retrospective review of 799, 449 surgical patient charts, only $65 \%$ of the patients received a dose of acetaminophen on the day of surgery. This was compared to $97 \%$ that received an opioid on the day of surgery. The authors further found that an average of only $34 \%$ of the patients received two or more nonopioid analgesics the day of surgery, despite the recommendations from multiple healthcare organizations such as the American Association of Anesthesiologists, CDC, and the US Department of Health and Human Services that patients should receive multimodal analgesia for optimal pain management during the perioperative period (Ladha et al., 2016). 
Despite the many years this topic has been examined, there still remains a large variation between providers, institutions, and regions to whether patients receive a multimodal approach to their perioperative pain management. Some institutions and practitioners still rely on a unimodal opioid only treatment (Ladha et al., 2016). Practitioners must rely upon current research to guide their decisions about which analgesics to prescribe for their patients. There is no universal guideline for multimodal analgesia, instead there are research-based recommendations from different specialty organizations on pain management, with many focused on chronic pain. Each practitioner determines the appropriate treatment based upon the type of surgery, past medical history, any genetic variants, and many other variables to prescribe a personalized pharmacologic approach (Hsu et al., 2019). In May 2019, the Journal of Orthopedic Trauma published its best practice recommendations for acute pain in musculoskeletal injuries. These recommendations were developed by a panel of 15 experts in the field of orthopedics and pain management. They reviewed the literature and based on the grading system, developed their best practice recommendations. These recommendations were approved in December 2018 and include the use of NSAIDs, acetaminophen, gabapentinoids, immediate-release opioids, and to consider the use of local or regional blocks. Despite these best practice recommendations, they do state that the final determination on pharmacological agents used should be patient personalized pharmacologic approach (Hsu et al, 2019).

Based on the above information, it is appropriate to explore the clinical question, "Is there a difference in postoperative opioid requirements in the first 24 inpatient hours in adults over the age of 60 undergoing primary hip arthroplasty depending on the 
number of multimodal analgesics they received?" Next a review of the literature will be discussed. 


\section{Literature Review}

An extensive literature review was performed using the data bases CINAHL, Google Scholar, Medline, OVID, Cochrane, and Pub Med. The search utilized the key words multimodal analgesia, preoperative analgesia hip arthroplasty, and preoperative multimodal orthopedics. The search was limited to articles dated from 2015 through 2019. Recommendations for articles for literature included in a review is $2-3$ years for science and 10 years for humanities (Wolf, 2019). To expand on the available research the date was extended to 2015. The websites that were utilized were the CDC and the US Department of Health and Human Services. The comprehensive literature review included opioid crisis, opioid epidemic, postoperative narcotic usage, acetaminophen perioperative, Celebrex, Gabapentin, total hip arthroplasty, and pain pathways.

\section{Prescription Opioid Epidemic}

The World Health Organization (WHO) developed the Pain Ladder or Analgesic Ladder in 1986. It was developed in response to guide practitioners in the treatment of cancer pain. At that time many cancer patients were undertreated for their pain (Singh et al., 2016). Narcotics were not administered freely, as it was believed that these patients could never be made pain free. The WHO Pain Ladder was designed to illustrate a stepwise approach to pain management starting with nonopioid analgesics in the treatment of cancer pain. Although the ladder is considered by health care providers to be outdated due to the extensive knowledge we have about pain and pain receptors, it contributed to the philosophy of multimodal analgesia. It also helped to change the practice of treating cancer pain. Despite the statement that it was developed to treat cancer pain, not for management of acute or chronic pain, it has been used to help guide 
providers in the treatment of non-cancer pain. The ladder currently does not consider the many different analgesic treatments that are currently available and relies only on nonopioid and opioid pharmacologic agents.

\section{Figure 1}

\section{World Health Organization Pain Ladder}

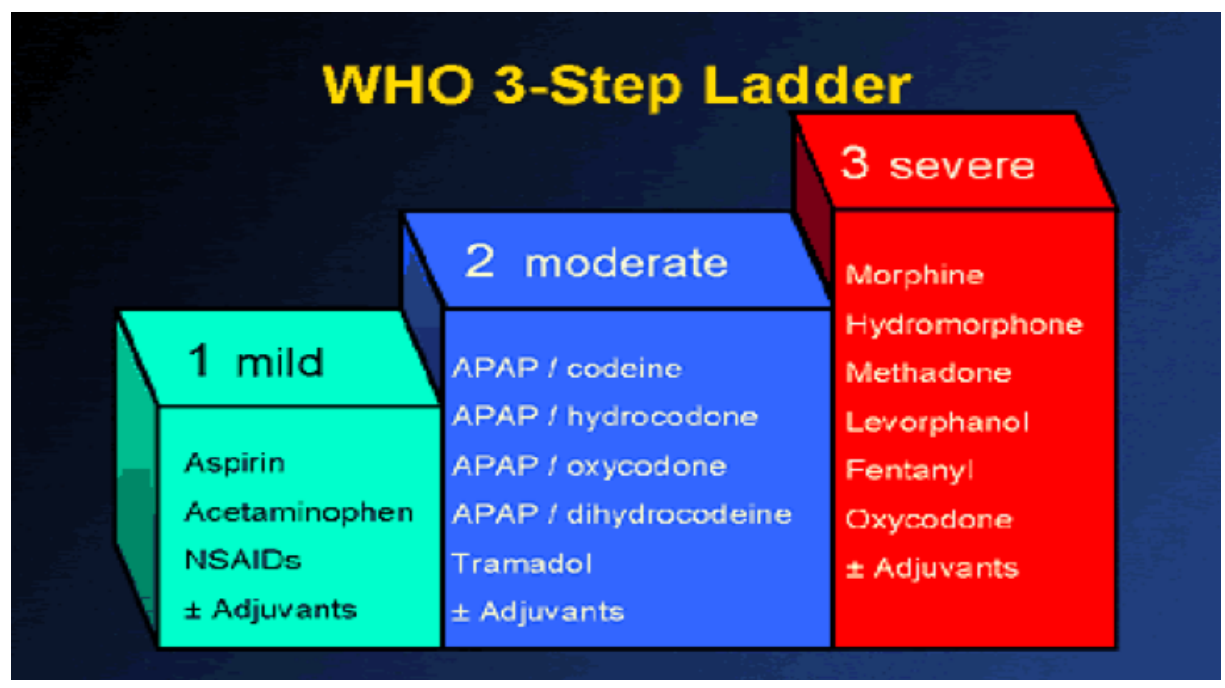

Note. The World Health Organization Pain Ladder (WHO, n.d.).

Following the WHO guidelines in the 1990s, patient advocacy groups urged for better pain control with patients. Specialty clinicians and professional groups advocated for more aggressive pain control. Misleading information was conveyed that opioids did not cause addiction, and in response, the amount of opioid prescriptions prescribed vastly increased (Trasolini et al., 2018). It was discovered in 2014 , that $40 \%$ of the world's supply of thebaine, the primary ingredient in two commonly prescribed opioids, oxycodone and hydrocodone, was utilized in the United States (Koepka et al., 2018).

Opioid prescribing has also been found to be influenced by patient satisfaction (Mistry et al., 2016). This is compounded by the fact that the primary survey used to collect patient satisfaction, Press Ganey, is now being utilized for reimbursement. A 
study by Mistry et al. (2016), examined how patient's pain control influenced their perception of the orthopedist, nursing staff, and satisfaction with the hospital overall. The results showed that pain control did not influence a hospital's overall rating; however, it did show a relationship with the patient's opinion of their nurses, physicians, and their general satisfaction with the hospital (Mistry et al., 2016). The authors also reported that in a survey by Zusman (2012), 27\% of physicians at that time reported their reimbursement was partially dependent on patient satisfaction scores. Some providers also reported that their employment was in jeopardy if they received poor patient satisfaction scores. The authors concluded that a patient's perception of their orthopedic surgeons was affected by their perception of how their pain was managed postoperatively. Those that perceived poor pain control rated their satisfaction with the orthopedic surgeon lower than those who perceived they had received adequate pain control.

Orthopedic surgeries are considered to be painful surgeries. According to the American Association of Orthopedic Surgeons, orthopedic surgeons are the third highest prescribers of opioid prescriptions (NYU Langone Health's Department of Orthopedic Surgery, 2019). Arthroplasty requires the orthopedist to cut deep into muscle tissue, and bone. Historically, it required large incisions with long hospital admissions, and assumedly more pain and discomfort. Since then, the procedure has changed, utilizing smaller incisions with shorter hospital length of stay. Despite smaller incisions, patients still reported substantial pain which led to a delay in initiating early physical therapy.

Orthopedists in the late 1990s were encouraged to treat the pain with narcotics, which also contributed to increased side effects. Presently, the majority of arthroplasty 
patients are 65 years and older. These patients are at risk for falls, and postoperative delirium along with other side effects related to opioid usage. The acknowledgement of these factors, along with the increased knowledge of pain pathways, contributed to a change in arthroplasty pain management (Trasolini et al., 2018).

Another contributing factor to the opioid epidemic is the availability of unused prescription narcotics in residential homes. In a systematic review, Bicket et al. (2017) found that many opioid pills remained untaken after orthopedic surgery. It has been suggested that decreasing the number of narcotic pills prescribed will decrease the availability of these opioids for illicit use and the number available for consumption, which may decrease the risk of a patient becoming a chronic user of opioids (Hsu et al., 2019). The CDC found that the rate of long-term opioid use was $6 \%$ one year later in patients who consumed 1 to 5 days of opioids. This rate increased to $13.5 \%$ when opioids were consumed for greater than eight days, and $29.9 \%$ when consumed for greater than 30 days (Shah et al., 2017). In a randomized sample of patients, joint pain was the most frequent reason cited for opioid being prescribed (Shah et al., 2017). To assist with this over prescribing of opioids, 15 states in the US limit the number days an opioid can be prescribed for an opioid naive patient for the treatment of acute pain. Three states limit the days to just five in opioid naïve patients. Some states have entrusted regulatory boards, such as the Department of Health or the State Board of Medicine, to regulate rather than the state deciding opioid prescription limits (Whitmore, \& Whisenant, 2019). Next the effects of undertreated pain will be discussed. 


\section{Effects of Undertreated Pain}

Chronic undertreated pain has been associated with the development of central sensitization (Vadivelu et al., 2014). Gabriel et al. (2019), discussed the concept of preemptive analgesia and central sensitization. Preemptive or preventative analgesia is defined as an analgesic given in the preoperative period prior to the incision to prevent the development of central sensitization. With the combination of the surgical incision and inflammatory process, preoperative pain can together contribute to the high reactivity of the central nervous system in chronic pain. Preventative analgesia can decrease postoperative pain levels and lessen the inflammatory response which can decrease the risk of central sensitization.

Total joint arthroplasty surgery of the lower extremity is one of the most common orthopedic surgeries performed. Historically, this type of surgery has been considered painful with poorly controlled postoperative pain management. Poorly controlled postoperative pain may lead to a delay in mobilization and postponed rehabilitation (Chen et al., 2015). A delay in beginning rehabilitation may lead to increased length of stay, readmissions, and increased cost of care along with decreased functional outcomes. To treat the pain, opioids especially high dose intravenous opioids in the form of Patient Controlled Analgesia (PCA) pumps, has become the treatment of choice (Parvizi \& Bloomfield, 2013). When patients are administered high dose opioids, they are at risk for developing increased adverse effects, along with impaired postoperative recovery, increased length of stay, and increased cost associated with treatment. Some of the common reported side effects of opioids are sedation, constipation, ileus, nausea, vomiting, urinary retention, pruritis, and respiratory depression. Respiratory depression 
in patients can lead to hypoxia which may cause additional side effects. Hypoxia, especially in older adults can lead to delayed wound healing, increased infection, myocardial infarction, and delirium (Parvizi \& Bloomfield, 2013). When epidural infusions have been used in place of PCA pumps with high dose opioids, these can also encounter side effects. Some significant side effects from epidural infusions are hypotension and urinary retention. Epidural infusions may also cause muscle weakness that can delay postoperative physical therapy which is necessary in the recovery phase of joint arthroplasty (Golladay et al., 2017).

One survey found that $80 \%$ of postoperative patients experience acute pain, and $86 \%$ of them rate the pain as moderate to severe (Apfelbaum et al., 2013). Postoperative pain that is left untreated can cause reactions to many of the body's systems. Untreated pain effects the gastrointestinal tract by causing decreased gastric mobility. Physical mobility is decreased in states of pain which places the patient at higher risk for developing deep vein thrombosis, muscle spasm, and muscle wasting. It affects the cardiovascular system by causing tachycardia, hypertension, and increased peripheral resistance. These effects may lead to myocardial ischemia, and increased risk of pulmonary emboli. Immobility due to pain can place the patient at risk for decreased cough and atelectasis. Prolonged pain effects the genitourinary system by causing urinary retention in patients. Psychologically, continual pain can lead to anxiety, fear, helplessness, and sleep deprivation. This may also lead to increased pain. In some cases, untreated pain may lead to a chronic pain state as result of central sensitization (Prabhakar et al., 2014). 


\section{Multimodal Analgesia}

Multimodal analgesia is defined as the use of two or more pharmacologic analgesics from different pharmacologic classes (Kehlet, \& Dahl, 1993). This allows for the use of less narcotic medication at a lower dosage, potentially decreasing adverse effects. By utilizing multimodal analgesia, it allows for genetic differences in opioid metabolism, any opioid tolerance, and pain sensitivity that a person may have (Manworren, 2015).

The implementation of multimodal analgesia allows for the blockage of the pain sensation through the different areas of the nociceptive and neuropathic pathway. By administering medications that exert their action on different areas of the pain pathway (Figure 2), it can decrease or block the neural pathway. The combinations of medications work together to create a synergistic response to pain. When tissue damage occurs, the pain is sensed by the nociceptors in the body. Local anesthetics such as peripheral nerve blocks, are able to target the specific site where the damage has occurred. Neuropathic pain arrives from damage to the nerve itself. The gabapentinoids and NMDA receptor antagonists exert their effect on the nerve itself by decreasing the stimulation and providing pain relief (Pitchon et al., 2018). By targeting different areas of the pain pathway, the patient achieves more effective pain control. This in turn leads less opioid consumption and fewer side effects (Parvizi \& Bloomfield, 2013). 


\section{Figure 2}

Pain Pathway

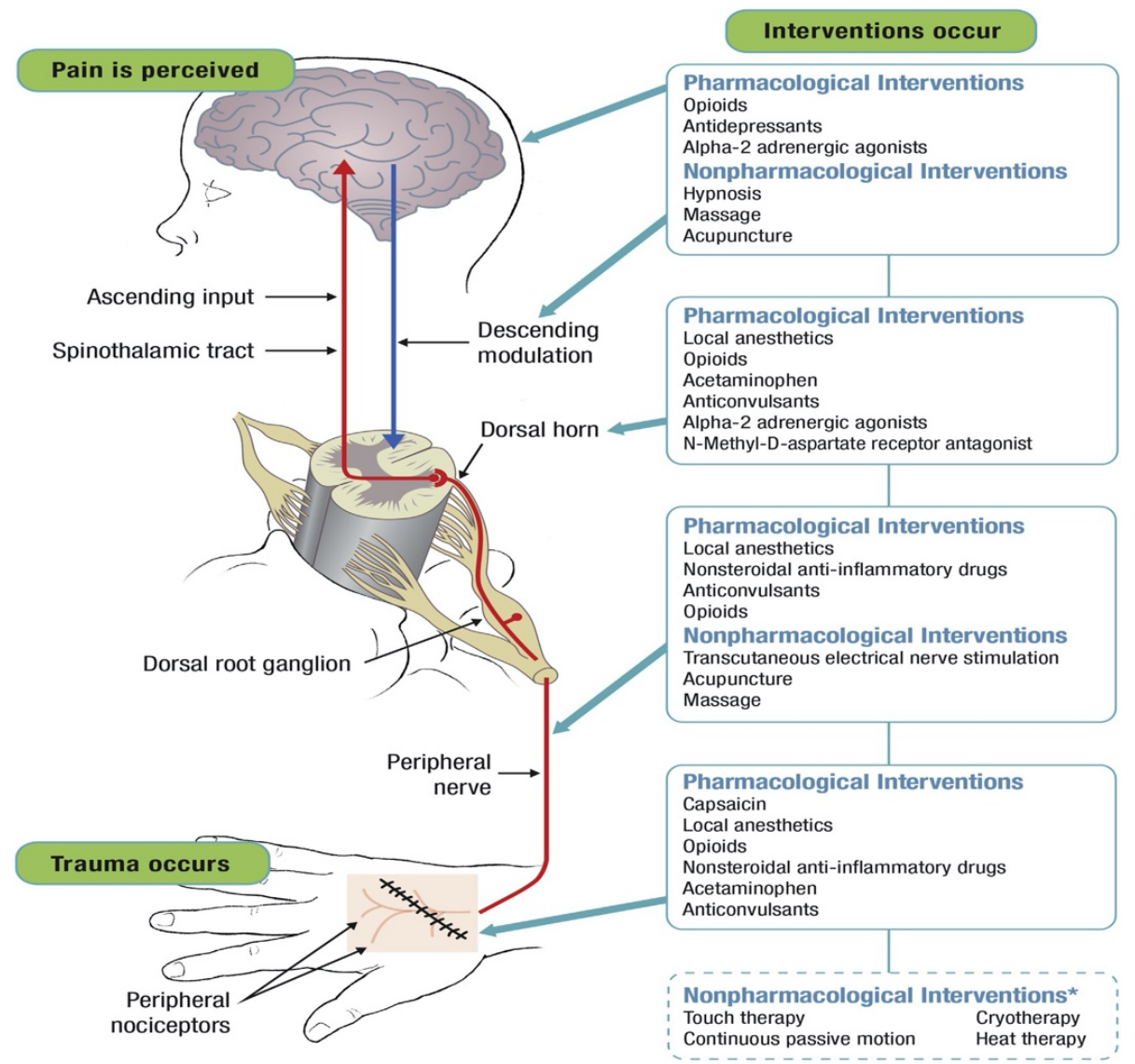

Note. Pain pathway and locations of analgesic target treatment areas (Manworren, 2015).

Preventative analgesia requires the use of several medications in the perioperative period. These may include the administration of N-methyl-D-aspartic acid (NMDA) receptor antagonists, epidural analgesia, neostigmine, ketamine, gabapentin, lidocaine, NSAIDs, and acetaminophen. All of these medications used in different combinations have been found to provide preventative analgesia when administered using a multimodal modal technique versus a unimodal technique. It allows for an increased length of analgesic effect which reduces sensitization caused by prolonged exposure to pain (Vadivelu et al., 2014). 
The American Society of Anesthesiologist Task Force on Acute Pain Management in 2012 recommended the use of certain medications for multimodal analgesia, although they state that every patient requires an individual approach. The list is meant as a guide not a checkoff. The key analgesics are acetaminophen, non-steroidal anti-inflammatory drugs (NSAIDs), gabapentinoids, opioids (short and long acting), spinal/epidural analgesia, regional nerve blocks, and local anesthetics (Stevenson et al., 2018). In the operating room suite, the use of ketamine, dexamethasone, magnesium, and dextromethophan are also included in the list of multimodal analgesics (Gabriel et al., 2019). The administration of acetaminophen and NSAIDs prior to opioids are highly recommended and encouraged as a primary intervention whenever possible. The use of regional analgesia (nerve blocks) has shown that by decreasing the nociceptive input it decreases the endocrine stress response and the pro-inflammatory response. This allows for increased tissue perfusion, decreased insulin resistance, and will improve a patient's coronary perfusion (Tornero et al., 2017). The most frequently administered analgesics will be discussed.

\section{Acetaminophen}

The cornerstone analgesic is acetaminophen. It has been shown to be safe and effective in the elderly population. It has an antipyretic, pain reducing effect, but it does not provide any anti-inflammatory effects (Harvard Health Publishing, 2018). The exact mechanism of action is believed to be related to a cyclooxygenase (COX) inhibition, or a change created to the endogenous cannabinoid system. It has minimal side effects, however there is a safety concern related to a dose dependent hepatotoxicity (Gabriel, et al, 2019). It has a smaller safety window compared to the NSAIDs. Large doses of 
acetaminophen can lead to liver damage and possible liver failure. The recommended maximum daily dose for a healthy adult is $4000 \mathrm{mg}$ a day. Even when taken at this dose, it may cause liver damage in some adults especially if alcohol is consumed concomitantly (Harvard Health Publishing, 2018).

Acetaminophen is available in many forms, which makes it an easy to administer drug. The intravenous form increases cerebrospinal fluid bioavailability, two times that of oral, and a quicker increased blood brain barrier penetration (Politi et al., 2017). In an experimental study, Singla et al. (2012) found that the average time to achieve maximum concentration of intravenous (IV) acetaminophen was 15 minutes. The same dose administered orally achieved maximum concentration levels in 45-75 minutes. The group that received IV acetaminophen also displayed higher cerebrospinal concentration than the group that received oral acetaminophen.

A study by Politi et al. (2017), compared IV versus oral acetaminophen as part of their institutions multimodal perioperative pain plan in patients who underwent total joint arthroplasty. The patients were divided, and each group received acetaminophen 1 gram prior to surgery and a scheduled dose every six hours after. Acetaminophen was administered for 24 hours. The visual analog scale (VAS) and the number of narcotics administered, was collected every 4 hours. The authors found that the 24-hour dosage of narcotics administered was equal in both groups, along with their 24-hour VAS scores. The only difference noted was in the 0-4 hour interval, where the VAS score was higher by one point in the group that received the oral acetaminophen, however the actual narcotic dosage administered in that time showed no significant difference. An approximate cost of 1 gram IV acetaminophen was $\$ 33.05$ versus $\$ 0.05$ for 1 gram of oral 
acetaminophen. Due to the cost, and lack of significant opioid consumption difference, the authors recommend oral acetaminophen versus IV whenever possible.

In a randomized blinded study (Westrich et al., 2019), IV acetaminophen was compared to oral acetaminophen in patients undergoing total hip arthroplasty. The groups either received 1 gram of IV acetaminophen with oral placebo, or 1 gram oral acetaminophen with IV placebo 30 minutes after entering the recovery room. They continued with this administration combination every six hours after the first dose until three days postoperative, or until discharge if that was earlier than three days. In this study, the patients were not prescribed any standing opioid orders. All opioids were ordered on an as needed basis. The authors found that there were no significant differences in pain scores, opioid side effects, or opioid administration between the two groups (Westrich et al., 2019).

In a randomized double-blind study, the administration of IV or oral acetaminophen was evaluated as an adjunct to a multimodal analgesia plan for patients undergoing total knee arthroplasty. The patients were divided into three groups; a placebo group, a group that received 1 gram of IV acetaminophen in the recovery room, and a final group that received 1 gram of oral acetaminophen in the recovery room. The authors evaluated the pain scores, and narcotic consumption for the first 24 hours amongst the three groups. In this study, investigators observed no significant difference amongst pain levels or narcotic consumption amongst the groups. The authors concluded that other studies had analyzed administration in the preoperative period and continued the administration of acetaminophen throughout the first 24 hours. In this study it was a onetime administration in the recovery room. The investigators concluded that 
acetaminophen administered preoperatively may affect the nociceptive receptors differently, which may bring about better analgesic effects (O’Neal et al., 2017).

\section{Anti-inflammatory medications}

Nonsteroidal anti-inflammatory drugs (NSAIDs) and cyclooxygenase-2 (COX 2) inhibitors provide an antipyretic and analgesic effect. They also have an antiinflammatory effect in which they inhibit prostaglandin production. This inhibitory effect can last through the intraoperative period into the postoperative recovery period. They block pain receptors peripherally in the ascending pain pathway, and centrally in the spinal dorsal horn. There they stop prostaglandin production via COX 2. They also stimulate the areas that encompass the descending inhibitory pain cascade. NSAIDs block both $\operatorname{COX} 1$ and $\operatorname{COX} 2$, whereas celecoxib and rofecoxib are inhibitors of COX 2 only (Gupta \& Bah, 2016).

There are concerns regarding potential adverse effects of NSAIDs. Their use is limited in the elderly and patients who have preexisting renal disease. Risks associated with the NSAIDs are cardiovascular, gastrointestinal, and renal. NSAIDs have traditionally been held prior to surgical procedures due to the risk of increased bleeding. Despite some reported risks, studies have shown no increased risk in cardiovascular incidents or bleeding when given in the acute postoperative period (Gupta \& Bah, 2016). The side effects from NSAIDs are due to prostaglandin synthesis. COX 2 inhibitors have less effect on the coagulation cascade, which make them favorable in the perioperative period for most surgeries (Stevenson et al., 2018). In patients undergoing colonic resections, the administration of NSAIDs and COX-2 inhibitors should be given with caution. There have been a few studies that have shown a significant increase in 
anastomotic leak when NSAIDs were given. This rate decreased when the medications were discontinued (Gupta \& Bah, 2016).

In a study by Chen et al. (2015), celecoxib was examined in elderly patients undergoing total hip arthroplasty. Patients were given a placebo, or a $400 \mathrm{mg}$ dose of celecoxib one hour preoperatively. Following the preoperative administration, the patients were then given the placebo, or $200 \mathrm{mg}$ of celecoxib every 12 hours for five days. The results showed that despite no statistical difference in VAS score, the group that received celecoxib was able to ambulate earlier than the patients who received the placebo. The celecoxib group consumed significantly less opioids than the control group did. There also was no statistical difference in intraoperative or post-operative blood loss between the two groups (Chen et al., 2015).

A comparison study by Onda et al. (2016), examined the effect on postoperative pain after administering either celecoxib, loxoprofen, or acetaminophen. Patients enrolled underwent arthroscopic knee surgery with removal of an internal fixator. The patients received either celecoxib $400 \mathrm{mg}$ for the first dose (200 $\mathrm{mg}$ the following doses), loxoprofen $60 \mathrm{mg}$, or acetaminophen $600 \mathrm{mg}$ in the postoperative period with the first dose given three hours after completion of surgery. Subsequently, the patients continued on the medication for 48 hours. The VAS at rest for all groups remained equal until the 24-hour mark in which the celecoxib and loxoprofen groups were significantly lower than the group that received acetaminophen. This result was also demonstrated at the 48-hour mark. When compared to the VAS with movement, the group that received the celecoxib had lower scores at all post medication time points. The researchers examined the patient's appraisal of their pain treatment outcome. Each group rated their pain 
management as excellent (no pain following treatment), good (significant improvement following treatment), acceptable (somewhat improved following treatment), and poor (no change or worse since treatment) 24 hours postoperative and 48 hours postoperative. The results showed no statistical difference in results between all groups at 24 hours postoperative. At 48 hours, the group that received celecoxib expressed greater satisfaction in their pain treatment compared to the other two groups. In the celecoxib group $47.2 \%$ responded that they received excellent results to their pain treatment (Onda et al., 2016).

Ketorolac is another NSAID that has antipyretic, analgesic, and anti-inflammatory effects. Its action inhibits the COX1 and COX2 enzymes. It is available in oral, IV, and intranasal (IN) forms. The drug was approved in 2010 for moderate to moderately severe pain for up to five days. The IN form was discovered to have the potency of morphine. Ketorolac IN is expensive. The price for five days of treatment was $\$ 1000$ (Pitchon et al., 2018).

Ketorolac is similar to other NSAIDs in the concern for delayed bone healing (Hassan \& Karlock, 2019). A study by Donohue et al. (2016), examined the healing rates of tibial and femoral fractures when ketorolac was administered. This retrospective chart review examined each fracture type divided into two groups. One group received ketorolac in the first 24 hours after the fracture, and the other group did not. Both groups displayed similar healing times, and similar rates of bone nonunion. In the group that received the ketorolac, only patients with a current history of smoking had delayed bone healing (Donohue, 2016). 


\section{Gabapentinoids}

The gabapentinoids include gabapentin and pregabalin. They are classified as anticonvulsant medications exerting an effect on the alpha2-delta unit in the calcium channels in the central nervous system. They decrease the discharge of neurotransmitters in the excitatory pathways (Gabriel et al., 2019). This inhibits pain transmission along with central sensitization (Schmidt et al., 2013). Adverse effects of gabapentinoids include sedation and respiratory depression (Gabriel et al., 2019). There are a multitude of studies using gabapentinoids as part of a multimodal analgesia regimen in the perioperative period. The studies all vary with timing, dosage, and medication; there are currently no specific recommendations for prescribing.

In a systematic review with meta-analysis by Fabritius et al. (2017), they examined 74 Randomized Controlled Trials (RCTs) and assessed the harm and benefit in the administration of gabapentin in procedure specific surgeries. The surgeries that were included were cholecystectomy, mastectomy, hysterectomy, thoracic surgery, spinal surgery, and orthopedic arthroplasty. These specific surgeries were chosen to represent a diverse patient population with various surgical procedures. A cholecystectomy is perceived as a minor surgical procedure that is often done as an outpatient surgery. Hysterectomy and mastectomy usually require a short stay and are considered to be associated with moderate amounts of pain. Thoracic surgery is associated with large amounts of pain and may require Intensive Care Unit (ICU) admission after the surgical procedure. The authors included orthopedic arthroplasty and spinal surgery to represent what they determined as surgical patients that preoperatively may represent patients with chronic pain. There was a total of 74 trials included in the RCT, however only eight 
trials were considered to have an overall low risk of bias. This included one trial from the cholecystectomy group, two trials from the hysterectomy group, two trials from the thoracic surgery group, and three trials from the orthopedic arthroplasty group. None of the trials done on patients having a mastectomy were considered low bias. The gabapentin dosages ranged from $300 \mathrm{mg}$ to $1800 \mathrm{mg}$. Morphine consumption at the 24hour mark was compared in 51 of the trials, but of those trials only seven were considered low bias. The authors found that in all six surgeries examined, there was a decrease in morphine consumption when gabapentin was administered. However, the authors did not specify whether the medication was administered preoperative or postoperative. They found that the trials all contained poorly documented serious adverse effects, with only half the trials reporting any adverse effects. The lack of adequate documentation led the authors of the systematic review to view the results as inconclusive.

A meta-analysis of randomized controlled trials by $\mathrm{Hu}$ et al. (2018), examined the effects of a single dose of pregabalin and gabapentin preoperative in relation to postoperative opioid consumption. In the 79 trials examined, they found the administration time varied from one hour before surgery to greater than one hour after surgery. The type of anesthesia administered also varied from spinal anesthesia to general anesthesia. The dose of pregabalin varied from $150 \mathrm{mg}$ to $300 \mathrm{mg}$, and the dose of gabapentin varied from $300 \mathrm{mg}$ to $1200 \mathrm{mg}$. The authors found that as the dose of pregabalin or gabapentin increased, the number of opioids consumed decreased. The greatest effect was observed with gabapentin $1200 \mathrm{mg}$ followed by pregabalin $300 \mathrm{mg}$. The study noted that high doses of pregabalin increased the rate of dizziness, but also 
decreased the rate of postoperative nausea and vomiting. High doses of gabapentin, 1200 $\mathrm{mg}$, were associated with increased rates of postoperative nausea and vomiting (Hu et al., 2018).

In a systematic review with meta-analysis, the authors examined the efficacy of administration of gabapentin or pregabalin in the preoperative period in patients undergoing total hip arthroplasty. The dosage of gabapentin ranged from $150 \mathrm{mg}$ to 1200 mg per day. In this study they found that the cumulative morphine dose was lower the first 24 and 48 hours on the pregabalin/gabapentin group, however there was no significant difference in their pain scores (Mao et al., 2016). Furthermore, a metaanalysis by Han et al. (2016), examined randomized controlled trials on total knee arthroplasty patients and the use of postoperative gabapentin. They evaluated narcotic consumption at 12 hours, 24 hours, and 48 hours postoperatively along with the patient's visual analog scale (VAS). They reviewed six studies which included 420 patients. The studies examined gabapentin versus a placebo preoperative, and gabapentin $300 \mathrm{mg}, 600$ $\mathrm{mg}$, and $900 \mathrm{mg}$ postoperative versus a placebo. The results showed a significant reduction in opioid consumption in the patients that received gabapentin in all of the hours examined. They did not see any significant difference in VAS scores between the groups during the time frame despite the reduction in opioid consumption.

In studies that compare the efficacy of gabapentin versus pregabalin, pregabalin was found to achieve longer postoperative pain-free periods than gabapentin. However, pregabalin can increase respiratory depression, so caution must be used in patients with obstructive sleep apnea (OSA) and the elderly population. Currently, only gabapentin is available in generic form, so there remains a cost difference between the two medications 
which could account for the limited studies evaluating the medications (Pitchon et al, 2018).

\section{NMDA receptor antagonist}

Ketamine is a N-methyl-D-aspartate (NMDA) receptor antagonist that was created in the 1960 s to be utilized as a general anesthetic. It provides its analgesic effect via the NMDA receptors. At subanesthetic dosages, it helps to control central sensitization and decreases opioid induced hyperalgesia. This is useful because it decreases opioid tolerance and is effective in treating patients with an opioid tolerance (Gabriel et al., 2019). Some animal studies have shown that NMDA antagonists can possibly reverse opioid induced tolerance along with the prevention of developing an opioid tolerance (Pitchon et al., 2018). Due to its effectiveness with opioid tolerant patients, it is now becoming part of the treatment plan in many hospitals for the treatment of pain in individuals who are opioid tolerant (Gabriel et al., 2019).

Ketamine use has been limited outside the operating room due to its side effects. It can cause psychomimetic effects that include hallucinations, vivid dreams, dissociative feelings, diplopia, blurred vision, sedation, and dysphoria. Most psychomimetic

symptoms resolve immediately after the medication is discontinued (Gabriel et al., 2019).

In an article by Schwenk et al. (2016), they found that $95 \%$ of adverse effects went away with the discontinuation of the ketamine infusion. Ketamine does not cause respiratory depression and has been shown to provide some postoperative analgesia and decrease opioid consumption. It is recommended that ketamine be used cautiously in the elderly population (Pitchon et al., 2018). 
The use of ketamine in spinal surgery has been well studied, but the use of ketamine in joint arthroplasty has not been examined as extensively. In a study by Cengiz et al. (2014), they examined the use of low-dose ketamine infusion during total knee arthroplasty surgery. In the study, which received ethical approval from the Clinic Research Ethical Community of The Turkish Ministry of Health, one group was administered a ketamine infusion, and the other group a placebo saline infusion. The time to first request for pain medication was longer in the group that received ketamine. They also found that morphine consumption at postoperative hours 1, 3, 6, 12, and 24 was also lower in the ketamine group. The amount of morphine administered decreased by $45 \%$. The length of time spent in the recovery room was increased in the group that received ketamine versus the saline infusion group. Patients' VAS all decreased in the group that received the ketamine infusion. However, the authors found no statistically significant difference between groups regarding adverse effects.

\section{Peripheral nerve blocks}

The use of regional blocks and local anesthetic injections have increased in the last few years with the addition of ultrasound to guide the injections (Gabriel et al., 2019). Multiple studies show decrease length of stay (LOS) and decrease opioid consumption in patients who receive regional blocks. The concern regarding blocks is the risk for infection or bleeding. Some site-specific risks are pneumothorax for brachial plexus and truncal blocks, quadricep weakness and falls from femoral nerve blocks, and peripheral nerve injury (Gabriel et al., 2019).

In a study by Ellis et al. (2018), patients undergoing total knee arthroplasty were evaluated before and after implementation of a clinical pathway that incorporated femoral 
nerve blocks, adductor canal blocks, and multimodal oral analgesic protocols. The clinical pathway was implemented and evaluated in the three phases. The first phase patients received only spinal anesthesia and a femoral nerve block. The second phase included spinal anesthesia, but the femoral nerve block was replaced by an adductor canal block. In the final phase, patients received spinal anesthesia, the adductor canal block and an oral multimodal analgesic protocol that was the same for all patients.

The researchers found that length of stay (LOS) was decreased in the groups receiving the adductor block versus the femoral block, and the group that received the adductor block with the oral multimodal analgesics had the shortest length of stay by one day. The group in phase one, two, and three average LOS were 3.89, 3.26, and 2.12 days respectively. The group that received the femoral block, phase one, rated on average higher pain levels than those that received the adductor block. The difference in pain scores between the two adductor groups was deemed not clinically significant (Ellis et al., 2018).

Total opioid consumption between the three groups revealed that the group that received the femoral block required more narcotics than the groups receiving adductor canal blocks. There was only a slight decrease in narcotic consumption for the group that received the oral multimodal medications which was not statistically significant. None of the groups received a standing postoperative acetaminophen order. When the doses administered were compared, there was significantly less administration of opioids in the two adductor canal groups compared to the femoral block group. There was no significant difference between the two adductor canal groups. Femoral blocks contribute to greater postoperative quadricep weakness as compared to adductor blocks. In this 
study, there were no patient falls reported in the adductor canal groups. The group that received the femoral block sustained five falls, which was $5 \%$ of that study group. There was significant decrease in antiemetic administration in the adductor canal groups as compared to the femoral block group. There were no other statistical differences in adverse effects between all three groups (Ellis et al., 2018).

In a Cochrane Systematic review by Chan et al. (2014), the effect of femoral nerve blocks (FNB) in total knee arthroplasty in postoperative pain were evaluated. They first examined pain levels at rest and with movement in patients comparing FNB with a PCA (Patient Controlled Analgesia), FNB without a PCA, and PCA only. They found that patients reported less pain when an FNB was performed. It also resulted in less opioid consumption, and consequently less postoperative side effects such as nausea, vomiting, and sedation. When FNB was compared with an epidural block, patients reported greater satisfaction with an FNB. The patients who received a femoral block also had decreased opioid consumption in the first 24 hours. The authors found only a single study that compared FNB and oral analgesia. In this study the FNB group had significantly less pain than the oral analgesia group. Finally, investigators compared a continuous FNB to a single dose FNB. The continuous FNB offered greater analgesia and led to lower opioid consumption in the first 24 hours (Chan et al., 2014).

\section{Guidelines for Analgesics in Orthopedic Patients}

Guideline and recommendations are written to provide patients with the most current up to date treatment and best outcomes. Such guidelines offer evidence-based practice recommendations that may come from government agencies such as the National Institute for Health, or specialty professional organizations such as the Orthopedic 
Trauma Association. Some guidelines are written in response to certain situations or events. The Orthopedic Trauma Association developed pain management guidelines for acute musculoskeletal injuries in response to the opioid epidemic. Studies have found orthopedic surgeons represent only $2.5 \%$ of all physicians but prescribe $7.7 \%$ of narcotic prescriptions. In a study by Hooten et al. (2015), researchers found that $21 \%$ of all patients who receive their initial prescription for pain medication will continue to receive additional prescriptions, and $6 \%$ of those patients will become chronic users. In addition, the authors also observed that many opioids prescribed after orthopedic surgery are unused, possibly contributing to the increased availability of opioids in the public. The organization felt the need to begin changing prescription habits of orthopedic physicians (Hsu et al., 2019).

In 2012 the American Society of Anesthesiologists Task Force on Acute Pain Management published evidence-based guidelines for the treatment of pain in the perioperative period. They recommended the use of multimodal pain management for patients and the consideration of regional blocks whenever possible. They further recommended the use of COX2, NSAIDs, or acetaminophen as a scheduled, not as needed, medication unless there was a contraindication (American Society of Anesthesiologists, 2012). In 2015, the American Pain Society published recommendations for management of postoperative pain with input from the American Society of Anesthesiologists. The guidelines recommend the use of multimodal perioperative analgesia, with the recommendation that acetaminophen and NSAIDs are prescribed whenever not contraindicated. They further recommended the administration of celecoxib, and a gabapentinoid in the preoperative period. The use of oral medication 
was recommended over intravenous medication, along with the use of nerve blocks whenever possible (Chou et al., 2016). A study by Ladha et al. (2016), suggests that the administration of multimodal analgesia was dependent on certain factors. Researchers hypothesized from the data that these were the culture of the hospital and the physicians' preference. Their results indicated that multimodal analgesia had not been universally adapted. The authors did not examine reasons for the low usage but suggest that the variation in evidence to support multimodal anesthesia across different surgical procedures may contribute to the reluctance to implement the recommendations. They also felt that the number of providers each patient sees in the perioperative period can make it difficult to determine who is the responsible provider for prescribing perioperative analgesics (Ladha et al., 2016).

In May of 2019 the Orthopedic Trauma Association posted guidelines for pain management in acute musculoskeletal injuries (Hsu et al., 2019). The 15-member panel consisted of experts in the field of orthopedic trauma, pain management, or both. The goal was to produce guidelines and recommendations to treat acute pain in patients that sustained musculoskeletal injury. It was also created to be utilized by other orthopedic practices and specialties. The research was reviewed and the recommendations they provided received a grade based on the Grading of Recommendations Assessment, Development, and Evaluation Working Group (Grade, n.d.). This method is used to grade the quality of the evidence, which is based upon the design of the study, the actual number of studies done, the sample size in each study, and if the results remain consistent amongst the studies. The studies are graded as strong (benefits outweigh potential harm) 
or as conditional (benefits do not outweigh potential harm). The evidence is further classified as having low, moderate, or high- quality evidence (Hsu et al., 2019).

The panel recommended the use of multimodal analgesia versus unimodal analgesia for pain control as a strong recommendation with moderate quality evidence. They referred to multimodal analgesia as a balanced analgesia, incorporating the use of multiple analgesics in addition to nonpharmacologic interventions that would affect the central or peripheral nervous system along the pain pathway. The use of periarticular injections as an adjunct was strongly recommended with moderate quality of evidence. The panel recommended prescribing the lowest dose of opioid for the shortest amount of time as strong with high-quality evidence. The recommendation to avoid long-acting opioids for acute injury is strongly recommended with low quality evidence. NSAIDs in patients with fractures, operative or nonoperative, is strongly recommended based on low quality evidence. The use of NSAIDs has been contraindicated in the past due to the concerns with bone healing. The panel found the evidence conflicting but based on beneficial results from use of NSAIDs to relieve musculoskeletal pain, withholding it from the multimodal plan may be more detrimental to the patient's pain relief than taking the medication (Hsu et al., 2019).

For the operative treatment of fractures, the panel recommends local or regional block anesthesia in the multimodal pain control plan as strong recommendation with high quality evidence. The panel also provided recommendations for pain relief after musculoskeletal injury using multimodal medications that include NSAIDs, acetaminophen, gabapentinoids, and immediate release opioids (Hsu et al., 2019). Whereas, the recommendations from the panel after a major musculoskeletal injury 
procedure, such as fixation of a long bone fracture, are the following for postoperative inpatient analgesia: ketorolac $15 \mathrm{mg}$ IV for five doses followed by ibuprofen every eight hours, gabapentin $100 \mathrm{mg}$ TID, acetaminophen $500 \mathrm{mg}$ every 12 hours, and oxycodone every four to six hours as needed for pain. The recommendations include hydromorphone $1 \mathrm{mg}$ IV every three hours prn for severe breakthrough pain. The panel also strongly recommends psychosocial interventions for patients who experience greater than expected or persistent pain. They also state that the use of these guidelines is not intended for the management of chronic pain (Hsu et al., 2019).

A recent retrospective study examined sleep apnea patients who received nonopioid analgesia (Cozowicz et al., 2019). The authors reviewed 181,182 patients' chart who underwent total hip or knee arthroplasty. Patient medical records were examined to determine if nonopioid analgesic modes were utilized the day of surgery or the day after. The information was then categorized as one, two, or more than two nonopioids administered. The non-opioids were described as peripheral nerve blocks, paracetamol or acetaminophen, steroids, gabapentin or pregabalin, ketamine, NSAIDs, or COX 2 inhibitors. Administration of a COX 2 inhibitor was found to decrease opioid use by $12.6 \%$, and the use of an NSAID decreased opioid consumption by $11 \%$. The researchers found that with the increase number of non-analgesic modes given, there was a stepwise decrease in opioid dose and postoperative complications. The largest reduction in postoperative complications were decreased postoperative mechanical ventilation and postoperative admission to a critical care unit. The decrease in the odds for mechanical ventilation and critical care admissions also stepwise decreased with the administration of two or more non-opioid analgesics. The chance for gastrointestinal complications 
decreased by up to $35 \%$ with administration of two or more non-opioid analgesics. The results showed that the patients who displayed largest opioid reduction, were those who received COX 2 inhibitors or NSAIDs. The study also found a stepwise decrease in LOS associated with increase in multimodal administration. The LOS decreased by up to $11.8 \%$ with the use of multimodal analgesia (Cozowicz et al., 2019).

\section{Barriers to Prescription Guideline Adherence}

A qualitative study was performed using the Theory of Planned Behavior to explore the barriers to the implementation of guideline prescribing recommendations. This was a small study of general practitioners in primary care. Seven themes emerged from the interviews. First, physicians questioned the credibility of the evidence. If they felt the evidence was weak, irrelevant, or unconvincing they would conclude that there was not credible evidence. They also were concerned with how rapidly the guideline may change, and frequent changes in practice may make patients skeptical of the care they were receiving (Rashidian et al., 2008). Second, if the guidelines were written by a source that was deemed credible, such as national guidelines, they were more apt to implement them. The sources that were perceived as not credible were deemed as low quality and low credibility and were not apt to be followed. The physicians cited sources such as pharmaceutical companies and consultants as untrustworthy (Rashidian et al., 2008).

The third theme recognized was the presentation of the guidelines. If the guidelines were complex and complicated they were less likely to be followed. Complicated guidelines were felt to be confusing and difficult to understand. The 
guidelines that were deemed strong and simple were more apt to be followed, although not all practitioners agreed upon what was perceived as simple (Rashidian et al., 2008).

The fourth theme to emerge was influential people. The physicians stated that colleagues, and local consultants influenced their treatment guidelines. They described coming to shared agreements regarding patient care in meetings. These decisions may or may not be in accordance with current guidelines. Patient preference also influenced treatment. The perceived pressure from patients as an influencing factor in what was prescribed in some cases (Rashidian et al., 2008).

The fifth theme identified was organizational barriers. The physicians described the inability to obtain access to certain resources. Some examples given were access to equipment, and support in implementing electronic databases. They reported time to implement as a barrier to compliance. The time requirement to disseminate the information and train the staff was perceived as difficult due to limitations in work time. These time constraints inhibited their ability to implement new guidelines. Some physicians also cited cost as a factor. If some of the new guidelines implemented new medications that were costly, that may deter the physician from following the guidelines or recommendations. They state they are under scrutiny to adhere to a prescription budget, and the cost of some newer medications exceed that budget (Rashidian et al., 2008).

The sixth common barrier was disease characteristics. The group of physicians evaluated were primary care physicians, and they felt that diseases that displayed complex characteristics, that guidelines were not helpful. The physicians had limited roles in treating these patients, and they had concerns in using guidelines in conditions 
that they considered difficult to diagnose. In other conditions that they considered simple, they believed a recommendation or piece of evidence may prove to be more beneficial than guidelines (Rashidian et al., 2008).

The final theme that emerged was implementation strategies. The physicians were reluctant to follow guidelines when it was handed to them with the expectation that they must follow it. They also reported concern, that if some guidelines are misinterpreted adverse outcomes may occur. The feeling of not being involved in the creation of the guideline was also perceived as a barrier to adherence. Some physicians felt that if they had more local involvement in the writing and implementation, they would be more willing to incorporate the guidelines. (Rashidian et al., 2008).

Likewise, a systematic review by Slade et al. (2016), examined barriers to clinical guidelines for management of low back pain by primary care clinicians. A total of 17 articles met criteria for review. They found three consistent emerging themes. The first was that the clinicians relied mostly on their past experiences and judgement over clinical guidelines. They also reported that current practice among peers was more important than what the guidelines recommended. The second theme was to maintain the patientclinician relationship. They acknowledged that patient's expectations may not be in line with current recommendations, and that if they did not follow through on the patient's requests that their clinical ability may be doubted. The final theme noted was time. Clinicians felt that they were overwhelmed with the number of guidelines, and did not have enough time to read, and incorporate them into their practice. They also cited lack of access or waiting times for other specialists or services such as physical therapists as a factor for providing treatment not recommended in the guidelines. The overuse of 
diagnostic imaging was a common theme. Patients request imaging, even if diagnostic imaging is not recommended in the guidelines. Delays in the ability to have patients seen by specialists prompted the clinicians to get diagnostic imaging because it was faster rather than follow current recommendations.

In summary, studies have shown mixed results about which types of analgesics provide optimal pain relief while decreasing opioid consumption. However, studies do suggest that applying a multimodal analgesic approach provides better pain relief by targeting different areas on the pain pathway (Golladay et al., 2017). Evidence does support that untreated pain can cause physical and psychological distress and may provide a role in chronic pain syndromes (Prabhakar et al., 2014). In 2019, the Orthopedic Trauma Association published guidelines to help provide evidence-based recommendations for pain management in patients with acute musculoskeletal injury. This was in response to the increase publicity and recognition of the opioid epidemic. They recognized that several professional organizations and government organizations had already released guidelines for safe opioid prescribing, although they mainly concentrated on chronic pain and not acute pain. The goal was to supply physicians with best practice recommendations, but they emphasized that implementation should be individualized and administered by physician discretion (Hsu et al., 2019). 


\section{Theoretical Framework}

Pain is a symptom that has a cascading effect on multiple systems in the body. Pain may originate from many different sources. The Theory of Unpleasant Symptoms (TOUS) is based on the premise that multiple symptoms can occur together (Lenz \& Pugh, 2013). The theory was created by four nurse researchers who began by independently researching the character and experience of different symptoms. Their shared perspectives and close proximity to each other allowed for a collaboration of information on their research. They found that there were related concepts and commonalities with their independent findings on fatigue during labor and delivery, which led to the development of a framework to explain fatigue in childbearing situations. The second partnership was during the discussion of fatigue with chronic obstructive pulmonary disease and asthma. During their discussions they realized there were a number of similarities between the two symptoms. This unexpected spontaneous discovery led the researchers to collaborate to develop a middle range theory that was clinically based (Lenz \& Pugh, 2013). 


\section{Figure 3}

Theory of Unpleasant Symptoms

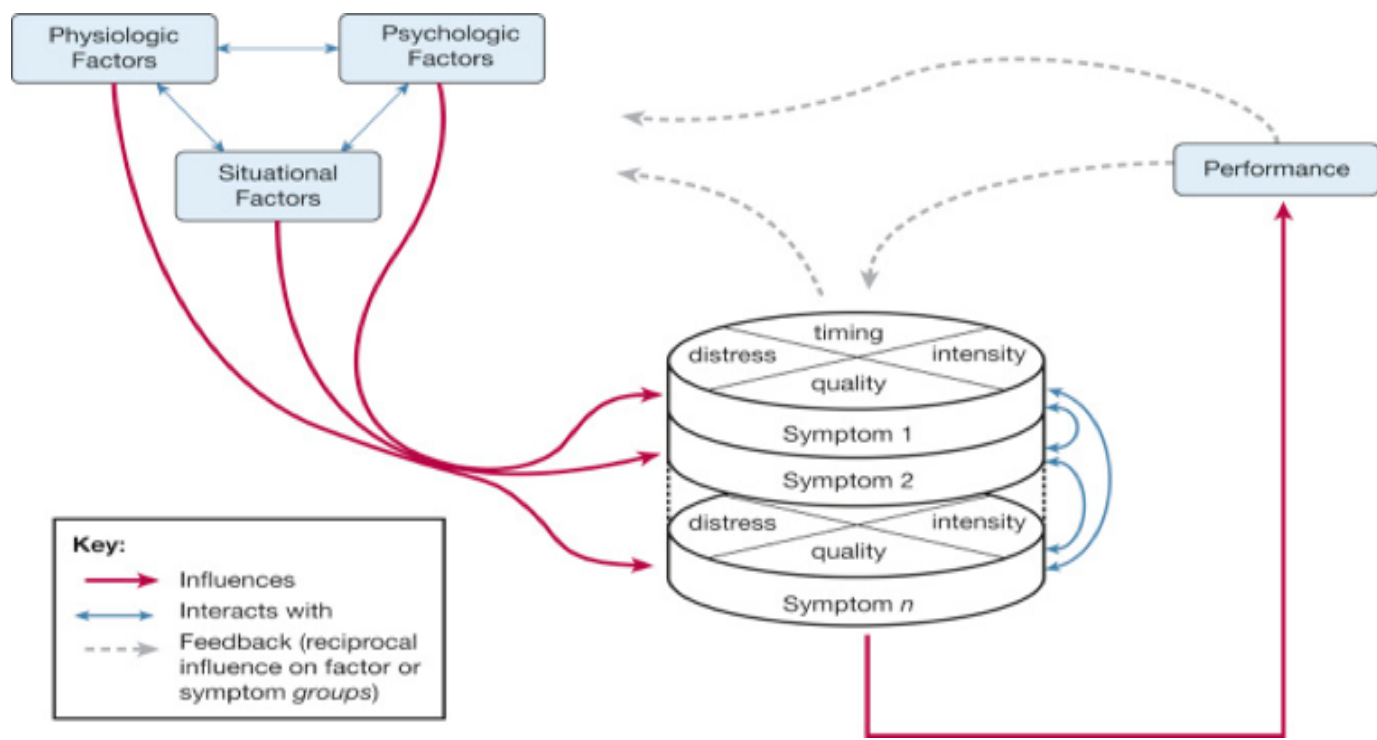

Note. The diagram of the middle range Theory of Unpleasant Symptoms (Lenz et al., 1997).

The TOUS is founded on three major concepts (Figure 3). The concepts are symptoms, influencing factors, and performance factors. The first concept symptoms is considered the central concept. Symptoms may be defined as a change in daily functioning which is reported by patients. The change that occurs may or may not be unpleasant and may or may not be an observable sign. Only the individual experiencing the change can truly measure it. The TOUS maintains that these symptoms can occur individually, in isolation, or with other symptoms (Lenz \& Pugh, 2013). In patients undergoing total hip arthroplasty, they are required to withhold any NSAIDs for a minimum of two weeks prior to surgery. Many of these patients have been on medication for years to help manage their arthritic pain. These patients arrive in the preoperative area and report significant pain. This increase in pain due to discontinuation of their 
medication may limit their ability to perform tasks that they were able to perform while on the medication. This along with the years of physical decline from their osteoarthritis is the "symptom" in the TOUS theory. The progressive decline through the years has caused many changes in their daily function.

The second major concept in TOUS is influencing factors. Influencing factors are elements that can change the way a symptom is experienced. Three factors have been identified that are considered most relevant. They are psychological, physiological, and situational factors. The combination of all three factors can result in a more intense symptom than just one or two of them combined. How a symptom is experienced is relevant to these specific factors, and how influential each factor is can be variable between individuals (Lenz \& Pugh, 2013). Hip arthroplasty is an elective surgery that is performed when a person can no longer perform their activities of daily (ADLs) due to pain and/or limitations in the joint. This may be due to collapse of the joint space or significant arthritic changes. Common physiological changes may be that a person is no longer able to walk upstairs or can no longer walk a distance of even five feet without severe pain. These changes may also include falls due to the collapse of the hip joint. The inability to be able to independently care for oneself can have significant psychological effects on a person, especially those that may be alone, or have limited assistance at home. Although this can be an extremely limiting situation for a patient, by medical standards the surgery itself is still classified as elective surgery. This means a patient can wait upwards of four months before they are able to have surgery. This wait period can cause increase stress on the patient prior to surgery. The loss of independence with the possible isolation from friends and family, and the waiting for surgery 
contributes to the influencing factors that change a way an event is perceived or experienced.

The final major concept is performance, which is the outcome concept of TOUS. The symptoms a person experiences can influence how a person is able to function or perform. There is an after effect to the symptoms that were experienced. This outcome may or may not affect the person's quality of life. The TOUS does not address quality of life as an outcome, but merely states that it is a feedback loop that has a reciprocal influence on the symptoms group (Lenz \& Pugh, 2013). The preoperative patient for total hip arthroplasty in some cases has waited months in discomfort with limited mobility. They are stressed, and anxious prior to arrival. Their success for an optimal recovery is dependent on their ability to actively engage in early/ immediate postoperative rehabilitation. By providing adequate preoperative education and medication, extreme pain and central sensitization can be prevented (Trasolini et al., 2018)

Patients who reported low postoperative pain levels have been shown to have a decreased morbidity rate and an improved postoperative recovery (Valencia \& Leopo, 2017). The ability to decrease anxiety and pain in the preoperative area may decrease postoperative anxiety and medication usage (Daltroy et al., 1998). The implementation of Enhanced Recovery Pathways (ERP) in patients undergoing total hip arthroplasty, which includes preoperative analgesia and early mobilization, has resulted in shorter LOS and satisfactory pain control (Ibrahim et al., 2013).

A patient undergoing a total hip arthroplasty who is anxious and in pain preoperative and postoperative may not be able to begin the necessary postoperative physical therapy immediately. Delay in activity predisposes patients to adverse 
physiological effects such as bed sores, deep vein thrombosis, myopathy, decreased respiratory function, and pneumonia (Soffin \& YaDeau, 2016). Whereas the patient who has less pain (symptoms), may be more willing to partake in therapy. Early mobilization in hip arthroplasty has been shown to decrease length of stay and increase patient satisfaction (Chen et al. 2012).

Patients in the perioperative period experience many unpleasant symptoms. These patients experience pain from their osteoarthritis, phlebotomy, and intravenous access necessary in the preoperative period. Many patients also experience a certain degree of anxiety, pain, fear, and apprehension prior to surgery. These symptoms can be placed in the Theory of Unpleasant Symptoms diagram (Figure 3), which illustrates how all of these combined can affect the outcome, or pain experience in the postoperative period.

In an article by Nguyen et al. (2017), the authors applied the Theory of Unpleasant Symptoms to the educational training of student nurse anesthetists. They explained that TOUS helps the students recognize the need to examine many aspects of a patient's symptoms. Nurses must scrutinize information relayed to them by patients and assess how some symptoms may influence other factors. Pain, anxiety, fear, and emotional stress are all unpleasant symptoms in the preoperative period. The student must examine these and provide measures to relieve the unpleasant symptoms which result in improved outcomes. During the postoperative period there may be increased pain, which if left untreated can cause physiological and psychological effects. The knowledge to apply multimodal analgesics can aid in alleviating some of the unpleasant symptoms. Another unpleasant symptom in the perioperative period is hypothermia. 
Patients lose heat quickly as a result of the cold operating room environment and anesthetics. The unpleasant symptom of being cold may lead to shivering and shaking. Application of warm blankets and warm intravenous fluids can help to maintain body temperature and provide comfort.

The final common unpleasant symptom in postoperative patients in nausea and vomiting. By assessing patients adequately in the preoperative period, appropriate preventative medications can be administered to relieve the unpleasant symptom. Risk factors such as prior history of postoperative nausea and vomiting, motion sickness, being female, and being a nonsmoker can place a patient at higher risk for nausea and vomiting after surgery. When identified as being at higher risk, appropriate anesthetic agents and medications can be administered to reduce the unpleasant side effect. By recognizing and understanding how all these unpleasant symptoms affect each other, the student nurse anesthetist is able to ease symptoms and provide optimal outcomes (Nguyen et al.,2017). The variables in the TOUS model align well with the overall aim of the proposed study that will exam pain management using a multimodal treatment approach with surgical adults who underwent total hip arthroplasty. 


\section{Method}

\section{Purpose}

The purpose of this project was to identify if there is a difference in postoperative opioid requirements in the first 24 inpatient hours in adults over the age of 60 years undergoing primary hip arthroplasty depending on the number of multimodal perioperative analgesics they received. The age of 60 was chosen based upon the findings from the American Joint Replacement Registry 2016 annual report that the median age of hip replacement in 2015 was 65.4 years old. The literature utilized for the review described the median ages of 62 to 68 years old for total hip arthroplasty. However, to increase the probability of obtaining an adequate sample size, patients' charts with a documented age of 60 years and older were selected for this study.

\section{Design}

The design was a retrospective chart review of patients who underwent primary total hip arthroscopy. Charts were categorized into five classes based on the multimodal analgesics received. Postoperative narcotic medication usage was converted to morphine equivalents to accurately examine total usage. Preoperative pain scores were compared to postoperative pain scores and compared against the different multimodal analgesia administered. Many patients arrive to the preoperative area with some degree of pain. By obtaining the preoperative pain level it can be determined if there was a reduction in overall pain level related to the number of multimodal analgesics administered.

\section{Sample}

The sample included 200 charts of adult patients who underwent elective primary total hip arthroplasty that met the inclusion and exclusion criteria. Sample size was 
determined by the estimate of patients who underwent total hip arthroplasty over a threemonth period at the clinical site and was elevated to provide an increased probability of detecting significant differences in the groups. Chart review included documented male and female patients age 60 and over with intact cognition. Exclusion criteria included patients with previous partial or total hip replacement on the same joint, currently on opioid medications for chronic pain (oxycodone, tramadol, morphine, oxycontin, or fentanyl), those with documented allergies to acetaminophen, and NSAIDs and acetaminophen, but an allergy or contraindication to NSAIDs alone did not exclude the patient. Further exclusions were patients with past medical history (PMH) of renal failure, liver disease, hemophilia, currently on suboxone or methadone, any documented alcohol or substance abuse disorder, and those with a diagnosis of neuropathic pain.

\section{Site}

The study site was the perioperative services and inpatient adult floors at a large tertiary care facility located in Rhode Island. The hospital is a 719 bed, non-profit, level 1 Trauma Center which is the primary teaching hospital for Warren Albert Medical School of Brown University in Providence Rhode Island.

\section{Ethical Considerations}

There were no ethical concerns identified. The medical charts reviewed were for discharged patients, and no charts of deceased patients were reviewed. None of the 18 Health Insurance Portability and Accountability Act (HIPAA) identifiers for protected health information were collected, and all charts reviewed were free of bias. The data used in the study were securely destroyed after completion of the study. 
The risk for bias was minimized with the extraction of data being collected through a computer program. A weakness of this study was the possibility that some information was missed and not included. It was not possible to examine all patient charts to see if to locate any missing data. Finally, the information in the chart is user dependent. Some information may not have been entered, such as last dose of a medication at home or pain scales.

\section{Procedure}

After approval to conduct the study was obtained from Lifespan and the Rhode Island College Institutional Review Board, a request was sent to Informational Services at the site to obtain the following information from patient records who underwent total primary hip arthroplasty: age, gender, allergies, past medical history, home medications (including last dose, date, and time taken), perioperative medications given, and inpatient medications administered in the first 24 hours. The above data was a report previously requested by the Department of Surgery at Lifespan which included patients who had a total hip arthroplasty and revision at this facility. Data collection was narrowed down to include only records from August 31, 2019 to December 31, 2016 per the approved IRB protocol (Appendix C). The above data was accessed by a computer-generated system that was placed under the "My Reports" section under Lifelinks, the hospital password protected program for the researcher to review. Data were screened for inclusion and exclusion criteria and the required data were extracted. Data collection began on January 25, 2020 and ended on March 13, 2020. No identifying data from the 18 HIPAA identifiers was collected. The data was placed in an Excel spreadsheet and kept in a password protected computer in a private office at the hospital facility. Data will be kept 
on an encrypted flash drive in a locked office and maintained for three years after the completion of the study. Only a Lifespan password protected computer will be used.

The medications that were obtained from the inpatient records included opioids, NSAIDS, acetaminophen, gabapentin, COX2 inhibitors (Celebrex), midazolam, diphenhydramine, and antiemetics (ondansetron). The opioid medications included fentanyl, hydromorphone, morphine, tramadol, Percocet, Vicodin, oxycodone, and oxycontin. The NSAIDs included ibuprofen, ketorolac, meloxicam, and diclofenac. Any NSAID, acetaminophen, COX2 inhibitor, or gabapentin taken at home within eight hours of surgery was included. The search also included whether a spinal or general anesthetic was administered and if a nerve block was performed. The patients' pain scores in the inpatient area was also collected.

For this study, the term preoperative referred to the time the patient arrived in the preoperative area until they are transferred to the operating room. The term intraoperative referred to their time in the operating room suite, and postoperative referred to the time that the patient was in the recovery room. The inpatient hours referred to the time the patient was considered recovered from anesthesia in PACU and could be transferred to an inpatient bed. Perioperative referred to the time the patient arrived in the preoperative area to the time the patient was discharged from the recovery room area or post anesthesia care unit (PACU), not including the time documented as Phase 2. When a patient had a prolonged recovery room time due to inability to obtain a floor bed, then inpatient time began when the patient was placed in Phase 2 in the recovery room. Phase 2 in the recovery room signifies that the patient had completed 
their immediate recovery room period, and had been cleared by the anesthesiologist for transfer, but there was no bed available for the patient.

\section{Data Measurement}

Data (specified above) were collected and measured using Microsoft Excel. To answer the research question, "Is there a difference in postoperative opioid requirements in the first 24 inpatient hours in adult over the age of 60 who underwent primary hip arthroplasty depending on the number of multimodal preoperative analgesics they received?" total opioid consumption and nonopioids prescribed in the first 24 inpatient hours along with pain levels were collected. A classification system was used to organize the data. Data were categorized as Class 0 (no medication), Class 1 (1 nonopioid medication), Class 2 (2 medications any type), Class 3 (3 medications any type), Class 4 (4 medications any type), and Class 5 (opioid only). Opioid consumption was converted into morphine equivalents among the groups using Global $\mathrm{RxPh}$ opioid conversion calculator (Global RxPh, n.d.). Postoperative pain scores were also compared between the groups.

\section{Data Analysis}

Descriptive statistics were used to analyze the data to determine whether a certain number of preoperative medications provided a greater reduction in postoperative opioid use and lower pain scale scores. Data collected from a total of 200 medical charts were analyzed using Microsoft Excel. The median, mode, and mean were evaluated and compared against the different number of multimodal analgesics administered. The total of all opioids administered were converted into morphine equivalents for comparison using the Global RxPh opioid conversion calculator (Global RxPh, n.d.). This opioid 
conversion tool was utilized in the Ellis et al. (2018) research article, along with other studies in the literature review. The data collected were analyzed and displayed using bar graphs. 


\section{Results}

A total of 418 charts were reviewed until 200 qualifying charts were identified. Females represented $54 \%(n=108)$ and males represented $46 \%(n=92)$ of the patients. The breakdown of patients per age group were as follows: $45.5 \%(n=93)$ were $60-69$ years old, $36 \%(n=72)$ were $70-79$ years old, $16 \%(n=32)$ were $80-89$ years old, and $1.5 \%$ $(n=3)$ were 90 years old or older. Analysis showed that $68 \%(n=136)$ of the qualifying 200 patients, did not receive any preoperative medications (Figure 4). Of the patients not receiving preoperative medications, $5.1 \%(n=7)$ had written orders that were never administered including one documented refusal. A total of $32 \%(n=64)$ patients received some type of medication in the preoperative period (Figure 5). These medications included one or any combination of the following; acetaminophen, COX2, gabapentin, oxycodone, or oxycontin (Figure 4). In examining the charts, it was noted that some patients received a dose of IV acetaminophen while in the operating room. These charts were further examined to determine if the dose of IV acetaminophen was administered prior to the first incision. The patients who received the IV acetaminophen prior to the first incision were included in the analysis accounting for $28 \%(n=18)$ of the patients who received preoperative medication. All of these patients were categorized into Class 1. 


\section{Figure 4}

Patients per Class

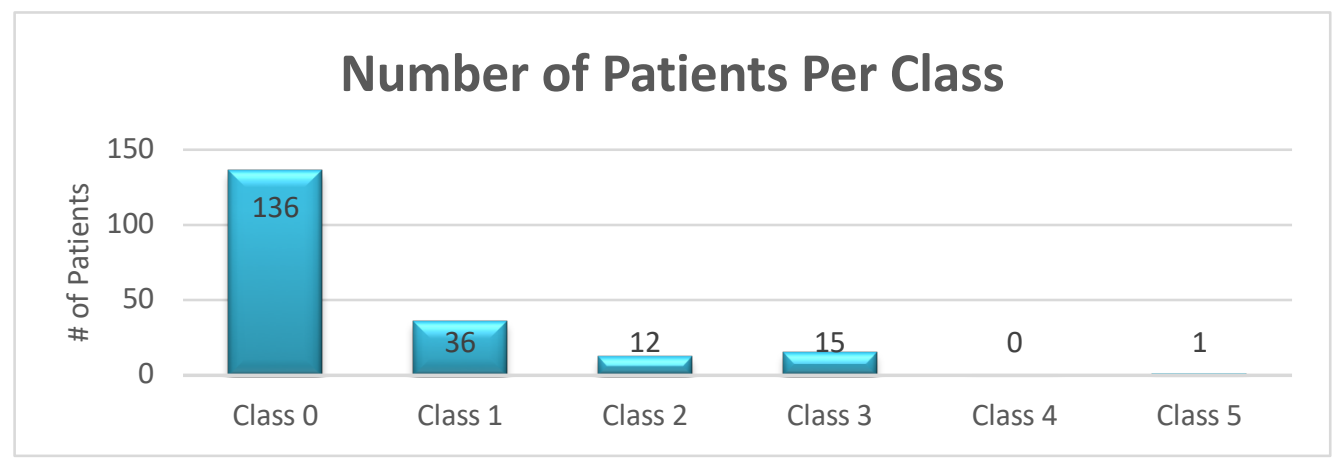

Note. Number of patients in each class of preoperative multimodal analgesia

\section{Figure 5}

Postoperative Administration by Class

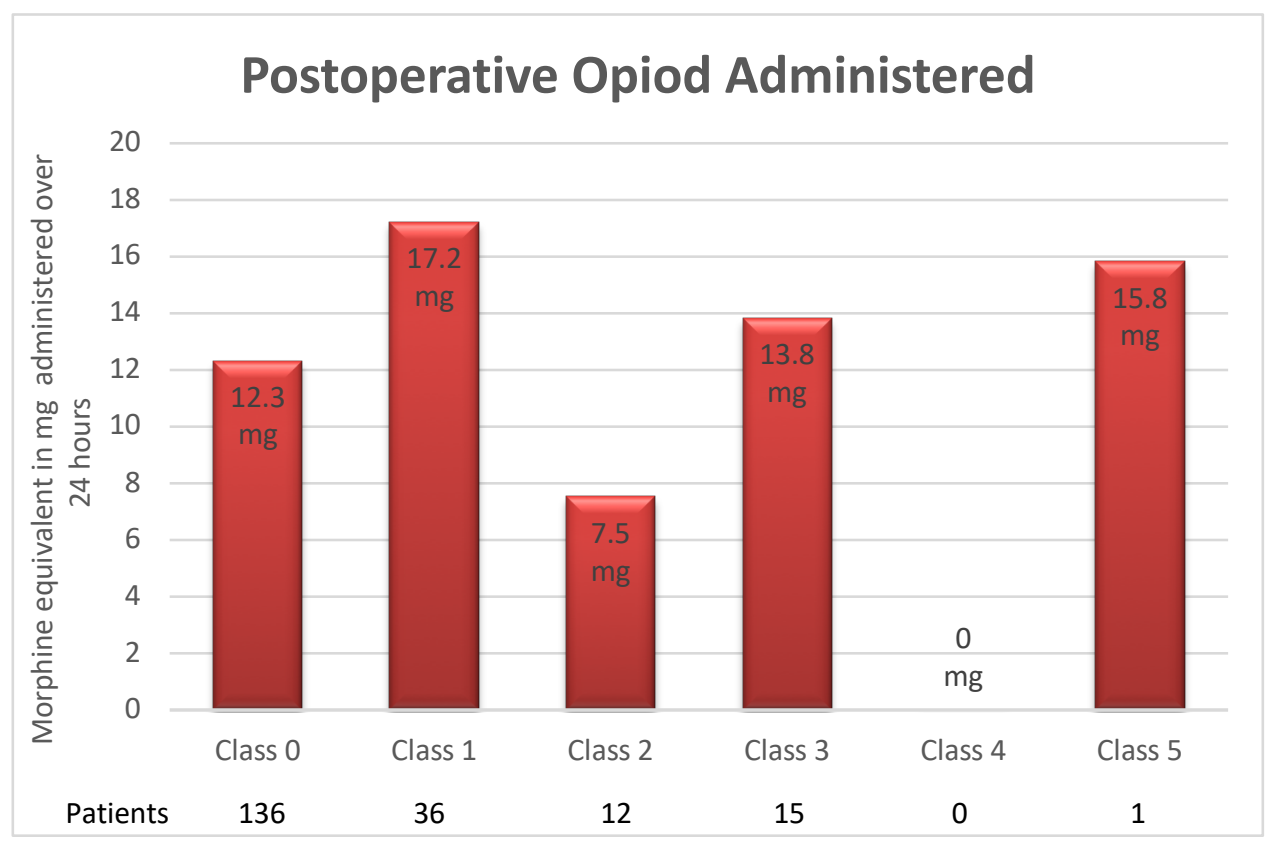

Note. First 24 -hour postoperative administration of opioids displayed as morphine equivalents.

Class 1 category comprised the largest number of patients. When the IV acetaminophen prior to incision was included, it comprised $56 \%(\mathrm{n}=36)$ of the patients 
who received a preoperative multimodal. Of those patients, $86 \%(\mathrm{n}=31)$ received acetaminophen and $13.9 \%(\mathrm{n}=5)$ received gabapentin preoperatively. The average narcotic usage in the first 24 hours for Class 1 was $17.6 \mathrm{mg}$ of morphine (Figure 6).

\section{Figure 6}

Class 1 Analgesic Breakdown

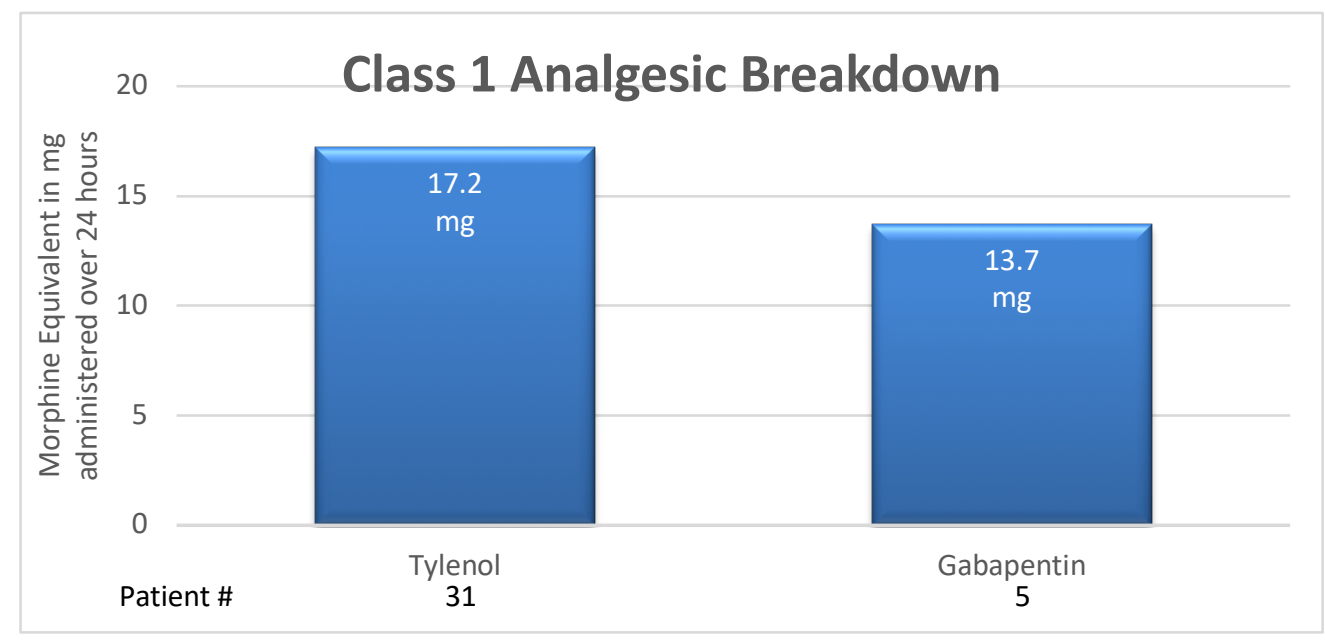

Note. Class 1 Preoperative multimodal analgesic breakdown by combination

The analysis showed that patients who received two preoperative multimodal medications, assigned as Class 2, required less opioid use in the first 24 hours postoperatively. Only a small sample, $18.7 \%(\mathrm{n}=12)$, received two preoperative medications. The average narcotic usage was $7.5 \mathrm{mg}$ of Morphine (Figure 7) in the first 24 hours postoperative. When those patients $(\mathrm{n}=12)$ were broken down by actual medications given, the number in each group was too small to make any conclusions about the combination of medications; 9 patients, 1 patient, 1 patient, and 1 patient in each combination (Figure 7). 


\section{Figure 7}

Class 2 Analgesic Breakdown

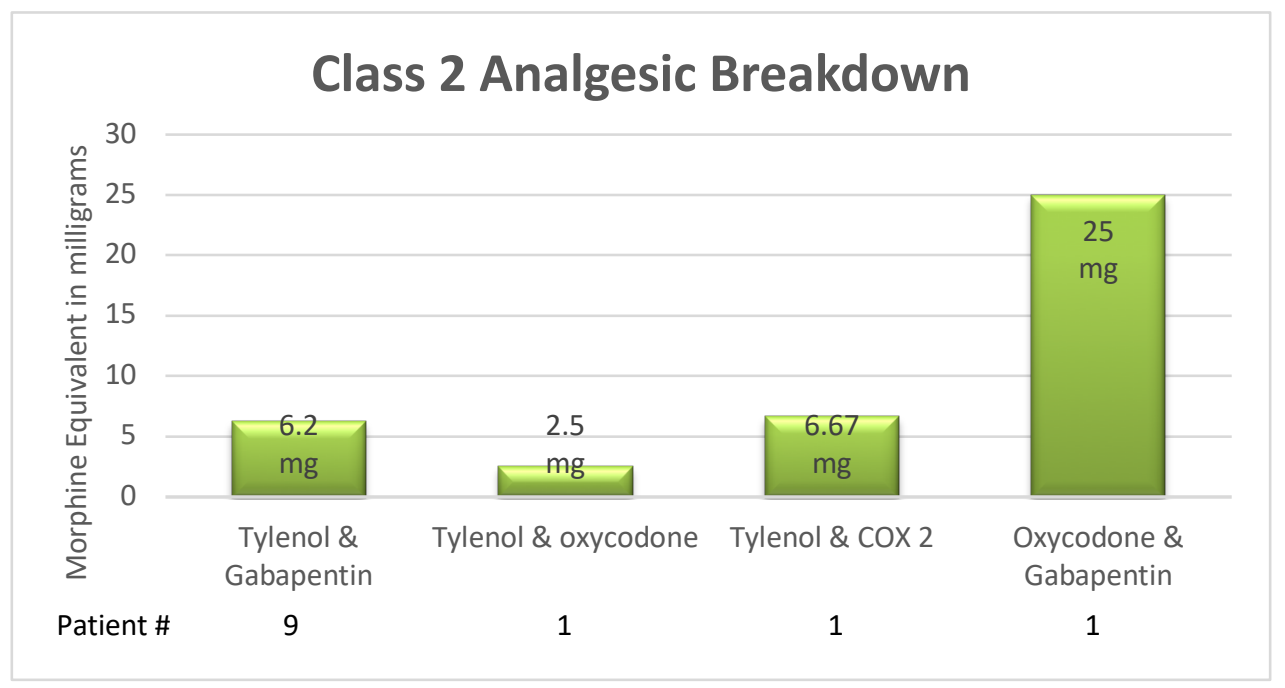

Note. Class 2 Preoperative multimodal analgesic breakdown by combination.

The Class 3 group 23.4\% $(n=15)$ received the second lowest consumption of morphine totaling $13.8 \mathrm{mg}$ of those that received preoperative medication. Despite the small number, when that group was broken down, there was a significant difference in the opioids administered between the groups. For example, patients who received Tylenol (acetaminophen), a COX2, and an oxycodone preoperatively had significantly lower opioid usage, compared to those who had Tylenol, gabapentin, and oxycontin, 5.4 mg and $19 \mathrm{mg}$ respectively (Figure 8). Given this represents only $9.4 \%(n=6)$ of the sample who received preoperative medications, it is too small to draw any meaningful conclusion. 


\section{Figure 8}

Class 3 Analgesic Breakdown

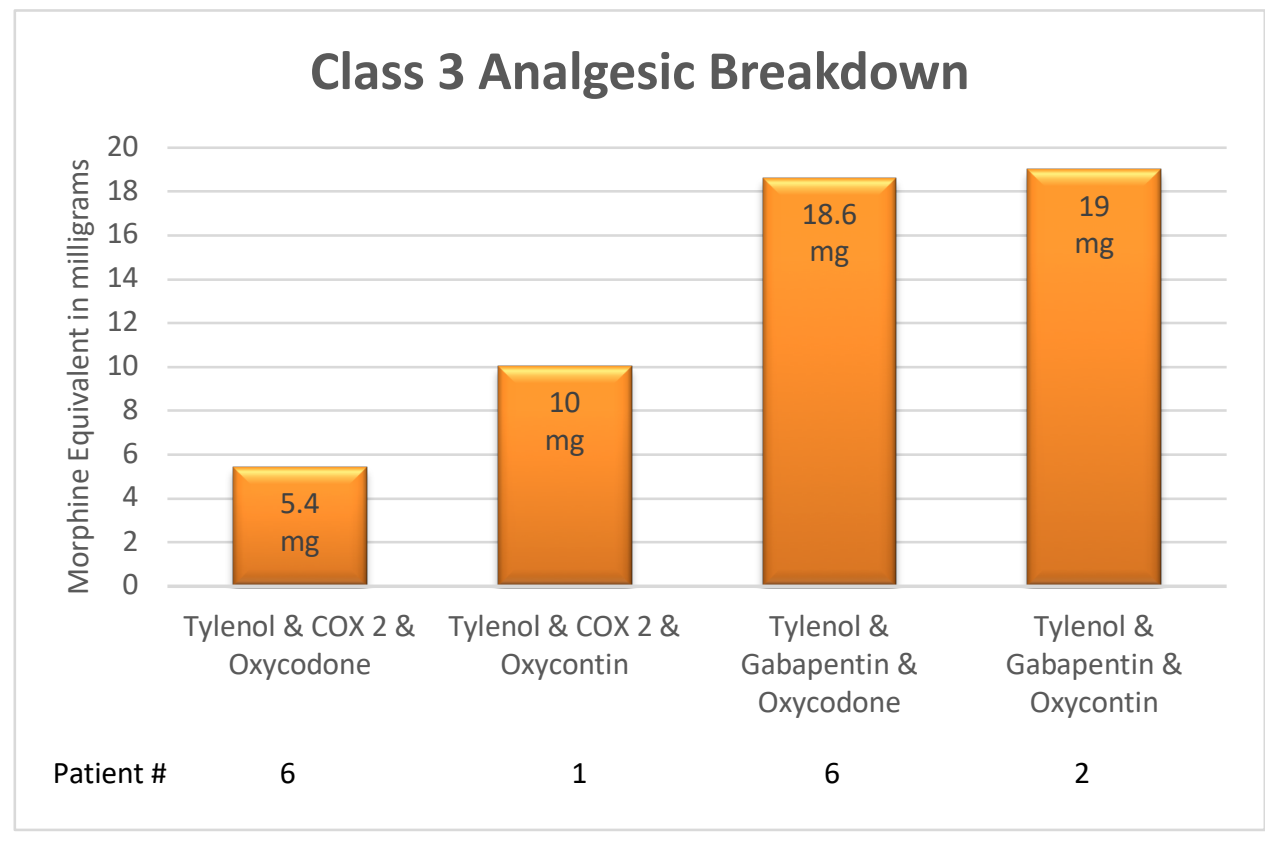

Note. Class 3 preoperative multimodal analgesic breakdown by medication combinations.

No patients in the qualifying group received 4 preoperative multimodals, so no data is presented in that column. There was only 1 patient who received an opioid only as a preoperative analgesic $1.6 \%(\mathrm{n}=1)$ of all patients receiving a preoperative medication. That patient's morphine equivalent was $15.83 \mathrm{mg}$ in the 24 hours postoperatively.

Roughly $10.5 \%(n=21)$ of the total patients received IV hydromorphone while in phase 2. Standing PACU orders for pain are for hydromorphone, with oxycodone not always being written as an option. Of those 21 patients, $14 \%(n=9)$ patients received a preoperative medication. Seven of the nine patients were categorized in class 1 , one in class 2 , and one was categorized in class 5 . Since the conversion from hydromorphone to morphine elicits a higher opioid usage than oxycodone, this may have affected some of 
the opioid usage totals showing a higher morphine equivalent than if they were not assigned to Phase 2 and were transferred when to an inpatient bed instead.

Pain scores were collected and analyzed on all patient groups postoperatively.

Pain scores were documented using the 0-10 Numeric Rating Scale Averages were calculated and are illustrated in Figure 8. Class 2 showed the lowest pain scores but had a standard deviation of 1.98. Conversely, Class 1 had the highest average documented pain score of 5. Those who received zero, three, and five preoperative analgesics had pain scores of 4.1, 4.4, and 4.2 respectively (Figure 9).

\section{Figure 9}

Inpatient Pain Scores

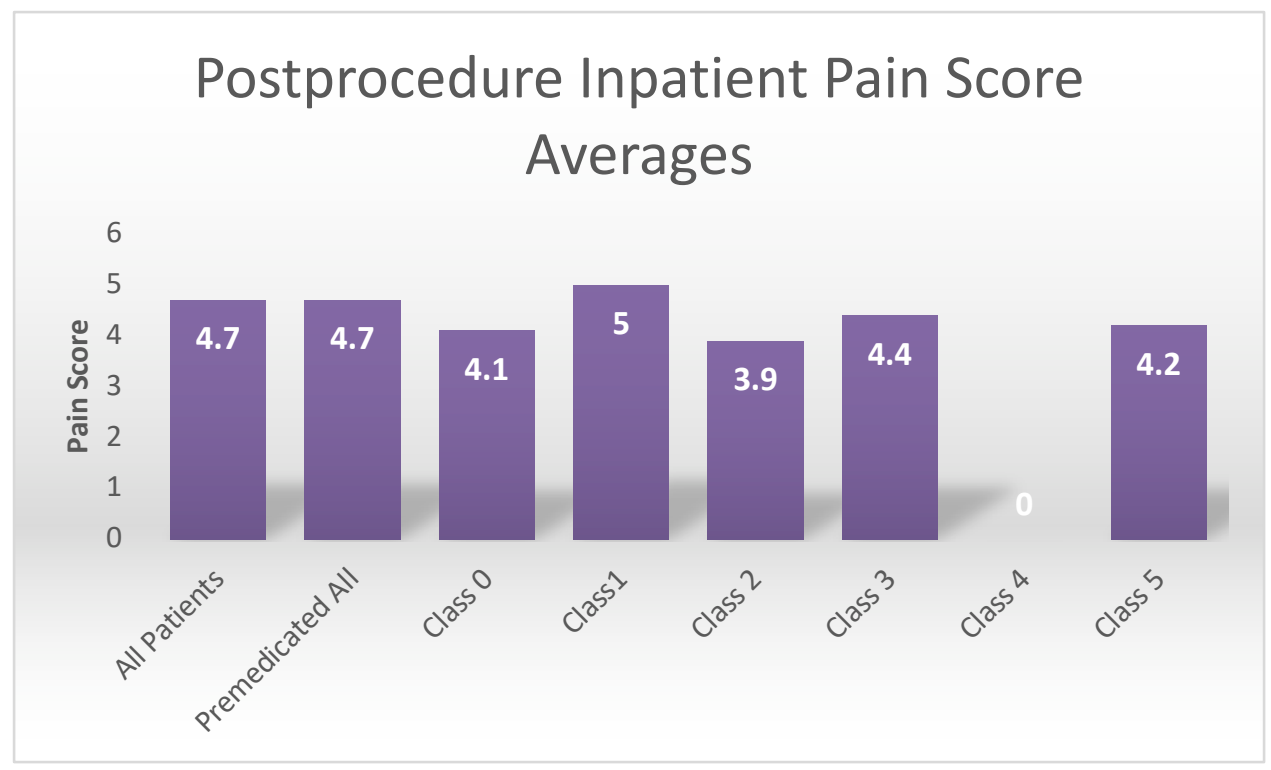

Note. Average inpatient pain scores by number of multimodal preoperative medications administered. 


\section{Summary and Conclusions}

The majority of the patients in this retrospective chart review who received a preoperative analgesia received a dose of acetaminophen $89 \%(n=57)$. In the retrospective chart review by Ladha et al. (2016), the authors found that only $65 \%$ of the patients received a dose of acetaminophen the day of surgery. In this retrospective chart review $97.5 \%(n=195)$ of the patients received a dose of acetaminophen the day of surgery. This included perioperative and inpatient medications administered the day of surgery. The patients who did not receive any acetaminophen $2.5 \%(n=5)$, three of the patients had an order but refused the medication. Only one patient was not prescribed postoperative acetaminophen, which included prn and standing orders. This aligns well with the WHO pain ladder that recommends the use of nonopioid medications as a first step (WHO, n.d.).

This retrospective chart review had limitations. A major barrier was obtaining information from the medical chart. There were inconsistent documentations of home medications, and the documentation of the first incision in the chart was not always consistent with the actual time of the incision, rather the time documented was the same time that was documented for the time the surgery ended. This excluded some charts in which IV acetaminophen was administered in the OR from being utilized, since the time of administration could not accurately be determined to be before the incision was made. These charts were then assigned as IV acetaminophen being administered was perioperative. Furthermore, there were cases in which the patient did not receive the prescribed preoperative medication, and other cases where the patient received preoperative acetaminophen and also received a second dose intraoperatively. These 
incidences possibly contributed to a smaller than actual patient population who received preoperative medications and could have skewed the study findings.

When reviewing the charts, it was observed that the type of medication and dose of medication prescribed were not totally dependent on a pain scale assessment. Pain management prescriptions are not universal to all providers patients. It was noted that some clinicians would prescribe for a nonopioid for pain rated as mild (0-4 on the numeric pain scale), whereas others may oxycodone in varying doses. Given the wide variations in dosing and types of medications prescribed, no definitive conclusion can be made regarding pain levels and the number of preoperative multimodals administered. A patient might have received two preoperative multimodals, but for pain considered moderate, may have been prescribed $10 \mathrm{mg}$ of oxycodone. Another patient with a different physician who also received two preoperative multimodals may be prescribed ketorolac for moderate pain.

The actual population of patients that received preoperative multimodal medication was small $32 \%(n=64)$ which does not allow for any conclusive results. However, the findings showed that when two multimodals were administered these patients used the least dose of opioids postoperatively and had the lowest pain scores compared to the other four classes. This suggests that by targeting the different pain pathways, a patient will experience less pain. Overall, as a combined group they were administered the smallest amount of postoperative opioid medications. When each class was broken down by medications, class 3 that included acetaminophen, a COX 2, and oxycodone required less opioids postoperatively. The percentage of preoperative 
medicated patients who received this combination, $9.4 \%(n=6)$, was very small to formulate a conclusive conclusion. It does however lend itself for further evaluation.

The charts that were reviewed were dated August 31, 2019 to the year 2016. The recommendations from the Orthopedic Trauma Association were not published until May 2019. This study could be replicated comparing the results from after the guidelines were published to the results before, to see if there has been a change in practice and a decrease in total opioid usage.

Overall due to the small sample size and large variability between opioid prescribing no conclusive results can be determined. An incidental finding while examining the results, was that the groups who consumed the lower amounts of postoperative opioids were more likely to have an order for around the clock acetaminophen and ketorolac prescribed postoperatively. When ketorolac was ordered as needed, the orders were prescriber dependent with variable pain scales used to determine administration. The medication administered was also user dependent. Some patients pain scale allowed for either an opioid or nonopioid for the same pain level, and there was no clear documentation which treatment option the patient requested or if it were determined by the nurse. This was an unexpected finding that was not part of the study. These possible findings suggest that expanding the study may yield more conclusive results 


\section{Recommendations and Implications for Advanced Nursing Practice}

The study revealed that despite the literature showing no analgesic advantage in this situation to the administration of IV acetaminophen versus oral acetaminophen, the IV version is still prescribed and is less cost effective. In this study, the percentage of patients who received a dose of either oral of IV acetaminophen during the perioperative period was $75 \%$, and the percentage prescribed around the clock postoperative acetaminophen was only $62 \%$. The WHO pain ladder recommends nonopioids as the first agent in pain control. Educating providers on the benefits of prescribing acetaminophen postoperatively with follow up evaluation could have a significant decrease in opioid administration postoperatively.

Advanced Practice Registered Nurses (APRN) are well positioned to educate staff, develop and implement Enhanced Recovery Pathways to optimize patient outcomes and decrease opioid use. The development of preoperative and postoperative surgery specific order sets could be developed that align with the WHO guidelines for pain management. The APRN is critical in the development of these due with their unique professional relationship with working with physicians and nurses. With a more consistent approach to medications ordered, medication and dosage ordered per pain, further studies can be done to determine what medication combinations provide the best pain relief while utilizing the least amount of narcotic. This study lends itself to further examination of the preoperative administration of multimodals, and examination of the effects on opioid usage with the administration of ketorolac postoperatively in pain management. 
The Theory of Unpleasant Symptoms can be applied when reviewing data and implementing changes. There may be the possibility that a COX2 preoperatively may decrease postoperative opioid usage. Implementing a COX2 order into the preoperative orders or prescribing it in the office to be administered at home prior to leaving for surgery, could give more information on its potential benefits. Premedicating falls under the physiological and psychological factors in the TOUS framework. The importance of alleviating some of the unpleasant factors is paramount in achieving optimal patient outcomes.

The APRN can promote evidence-based education to physicians and nurses. The basis is improving the care and the quality of life for these patients. Serving as a resource for staff and implementing education programs or leaflets for patients will enable them to better understand the goals of pain management. With additional research the APRN can identify barriers to achieving optimal pain management, and processes that resulted in optimal patient outcomes. 


\section{References}

American Society of Anesthesiologists Task Force on Acute Pain Management. (2012). Practice guidelines for acute pain management in the perioperative setting: an updated report by the American Society of Anesthesiologists Task Force on Acute Pain Management. Anesthesiology, 116, 248-273.

Apfelbaum, J. L., Chen, C., Mehta, S. S., \& Gan, T. J. (2003). Postoperative pain experience: results from a national survey suggest postoperative pain continues to be undermanaged. Anesthesia \& Analgesia, 97(2), 534-540.

Bicket, M. C., Long, J. J., Pronovost, P. J., Alexander, G. C., \& Wu, C. L. (2017). Prescription Opioid Analgesics Commonly Unused After Surgery. JAMA Surgery,152(11), 1066-1071. doi:10.1001/jamasurg.2017.0831

Cengiz, P., Gokcinar, D., Karabeyoglu, I., Topcu, H., Cicek, G. S., \& Gogus, N. (2014). Intraoperative Low-Dose Ketamine Infusion Reduces Acute Postoperative Pain Following Total Knee Replacement Surgery: A Prospective, Randomized DoubleBlind Placebo-Controlled Trial. Journal of the College of Physicians and Surgeons Pakistan,24(5), 299-303. doi:04.2014/JCPSP.299303

Centers for Disease Control and Prevention (CDC). (2019). Understanding the Epidemic. Retrieved from https://www.cdc.gov/drugoverdose/epidemic/index.html Centers for Disease Control and Prevention (CDC) WISQARS. (2019a). 1999. United States Unintentional Injuries- Poisoning All Ages, All Races, Both Sexes. Retrieved from https://webappa.cdc.gov/cgibin/broker.exe?_program=wisqars.dd_details10drill.sas\&_server=aspv-wisq1. cdc.gov\& service $=\mathrm{v} 8$ prod\&_port $=5098 \&$ sessionid $=$ bjTKyt 7 wP52\&type $=U \&$ 
$\underline{\text { dcause }=\text { Poisoning \&save causestring }=(\text { cause } 113 \% 20 \text { in } \% 20(210701)) \& \text { age } 1=. \& a}$ ge $=. \&$ save_yeartxt $=2017 \&$ save_stname $=$ United $\% 20$ States\&injtitle=Unintention al\%20Injuries\&agetitle=All\%20Ages\&save_racetxt=All\%20Races,\&save_sextxt $=$ Both $\% 20$ Sexes $\&$ totald $=\% 20 \% 20 \% 20 \% 2064,795 \&$ save state $=00 \&$ save race $=0$ \&save_sex $=0 \&$ save_ethnicty $=0 \&$ debug $=0$

Centers for Disease Control and Prevention (CDC) WISQARS. (2019b). Unintentional Injuries- Poisoning All Ages, All Races, Both Sexes. Retrieved from https://webappa.cdc.gov/cgibin/broker.exe?_program=wisqars.dd_details10drill.sas\&_server=aspv-wisq1.cdc.gov\&_service $=$ v8prod\&_port $=5098 \& \_s e s s i o n i d=D 8 Q t j B 8 w P 52 \&$ type $=U \&$ dcause $=$ Poisoning \&save_causestring= $=($ cause $113 \% 20 \mathrm{in} \% 20(210701)) \&$ age $1=. \& \mathrm{a}$ ge2=.\&save_yeartxt $=1999 \&$ save_stname $=$ United\%20States\&injtitle $=$ Unintention al\%20Injuries\&agetitle=All\%20Ages\&save_racetxt=All\%20Races,\&save_sextxt $=$ Both $\% 20$ Sexes\&totald $=\% 20 \% 20 \% 20 \% 2012,186 \&$ save_state $=00 \&$ save_race $=0$ $\&$ save_sex $=0 \&$ save_ethnicty $=0 \&$ debug $=0$

Chan, E., Fransen, M., Parker, D. A., Assam, P. N., \& Chua, N. (2014, May 13). Femoral nerve blocks for acute postoperative pain after knee replacement surgery. Retrieved July 3, 2019, from https://www.cochrane.org/CD009941/ANAESTH_femoral-nerve-blocks-acutepostoperative-pain-after-knee-replacement-surgery

Chen, A. F., Stewart, M. K., Heyl, A. E., \& Klatt, B. A. (2012). Effect of immediate postoperative physical therapy on length of stay for total joint arthroplasty patients. The Journal of Arthroplasty, 27(6), 851-856. 
Chen, J., Zhu, W., Zhang, Z., Zhu, L., Zhang, W., \& Du, Y. (2015). Efficacy of celecoxib for acute pain management following total hip arthroplasty in elderly patients: A prospective, randomized, placebo-control trial. Experimental and Therapeutic Medicine,10(2), 737-742. doi:10.3892/etm.2015.2512

Chou, R., Gordon, D. B., de Leon-Casasola, O. A., Rosenberg, J. M., Bickler, S., Brennan, T., ... \& Griffith, S. (2016). Management of Postoperative Pain: a clinical practice guideline from the American pain society, the American Society of Regional Anesthesia and Pain Medicine, and the American Society of Anesthesiologists' committee on regional anesthesia, executive committee, and administrative council. The Journal of Pain, 17(2), 131-157.

Cozowicz, C., Poeran, J., Zubizarreta, N., Liu, J., Weinstein, S. M., Pichler, L., . . Memtsoudis, S. G. (2019). Non-opioid analgesic modes of pain management are associated with reduced postoperative complications and resource utilization: A retrospective study of obstructive sleep apnea patients undergoing elective joint arthroplasty. British Journal of Anesthesia, 122(1), 131-140.

doi:10.1016/j.bja.2018.08.027

Daltroy, L. H., Morlino, C. I., Eaton, H. M., Poss, R., \& Liang, M. H. (1998).

Preoperative education for total hip and knee replacement patients. Arthritis \& Rheumatism: Official Journal of the American College of Rheumatology, 11(6), 469-478.

Donohue, D., Sanders, D., Serrano-Riera, R., Jordan, C., Gaskins, R., Sanders, R., \& Sagi, H. C. (2016). Ketorolac administered in the recovery room for acute pain 
management does not affect healing rates of femoral and tibial fractures. Journal of Orthopedic Trauma, 30(9), 479-482.

Ellis, T. A., Hammoud, H., Merced, P. D., Nooli, N. P., Ghoddoussi, F., Kong, J., \& Krishnan, S. H. (2018). Multimodal Clinical Pathway with Adductor Canal Block Decreases Hospital Length of Stay, Improves Pain Control, and Reduces Opioid Consumption in Total Knee Arthroplasty Patients: A Retrospective Review. The Journal of Arthroplasty,33(8), 2440-2448. doi:10.1016/j.arth.2018.03.053

Fabritius, M. L., Geisler, A., Petersen, P. L., Wetterslev, J., Mathiesen, O., \& Dahl, J. B. (2017). Gabapentin in procedure-specific postoperative pain managementpreplanned subgroup analysis from a systematic review with meta-analysis and trial sequential analysis. BMC Anesthesiology,17(85). Retrieved from doi $10.1186 / \mathrm{s} 12871-017-0373-8$

Gabriel, R. A., Swisher, M. W., Sztain, J. F., Furnish, T. J., Ilfeld, B. M., \& Said, E. T. (2019). State of the art opioid-sparing strategies for post-operative pain in adult surgical patients. Expert Opinion on Pharmacotherapy,20(8), 949-961. doi:10.1080/14656566.2019.1583743

Galloway, K. T., Buckenmaier, C. C., Gallagher, R. M., \& Polomano, R. C. (2011). Special report- War on pain: Multimodal and multidisciplinary therapy for pain management. American Nurse Today,6(9). Retrieved June 6,2019, from https://www.americannursetoday.com/multimodal-multidisciplinary-therapy-painmanagement/ 
Global RxPh. (n.d.). Opioid Conversions Calculator Equivalent Dosages- Advanced. Retrieved September 2, 2019, from https://globalrph.com/medcalcs/opioid-painmanagement-converter-advanced/

Golladay, G. J., Balch, K. R., Dalury, D. F., Satpathy, J., \& Jiranek, W. A. (2017). Oral Multimodal Analgesia for Total Joint Arthroplasty. The Journal of Arthroplasty,32(9), S69-S73. doi:10.1016/j.arth.2017.05.002

GRADE working group. (n.d.). Retrieved September2, 2019 from http://www.gradeworkinggroup.org/

Gupta, A., \& Bah, M. (2016). NSAIDs in the treatment of postoperative pain. Current Pain and Headache Reports, 20(62). doi:DOI10.1007/s11916-016-0591-7

Han, C., Li, X., Jiang, H., Ma, J., \& Ma, X. (2016). The use of gabapentin in the management of postoperative pain after total knee arthroplasty: A PRISMAcompliant meta-analysis of randomized controlled trials. Medicine,95(23). doi:10.1097/MD.0000000000003883

Harvard Health Publishing. (2018, October 23). Acetaminophen safety: Be cautious but not afraid. Retrieved from https://www.health.harvard.edu/pain/acetaminophensafety-be-cautious-but-not-afraid

Hassan, M. K., \& Karlock, L. G. (2019). The effect of post-operative NSAID administration on bone healing after elective foot and ankle surgery. Foot and Ankle Surgery. https://doi.org/10.1016/j.fas.2019.05.016

Hooten, W. M., St Sauver, J. L., McGree, M. E., Jacobson, D. J., \& Warner, D. O. (2015, July). Incidence and risk factors for progression from short-term to episodic or 
long-term opioid prescribing: a population-based study. In Mayo Clinic Proceedings (Vol. 90, No. 7, pp. 850-856). Elsevier.

Hsu, J. R., Mir, H., Wally, M. K., \& Seymour, R. B. (2019). Clinical Practice Guidelines for Pain Management in Acute Musculoskeletal Injury. Journal of Orthopedic Trauma,33(5), E158-E182. doi:10.1097/BOT.0000000000001430

Hu, J., Huang, D., Li, M., Wu, C., \& Zhang, J. (2018). Effects of a single dose of preoperative pregabalin and gabapentin for acute postoperative pain: A network meta-analysis of randomized controlled trials. Journal of Pain Research, 11, 26332643. doi:10.2147/jpr.s170810

Ibrahim, M. S., Khan, M. A., Nizam, I., \& Haddad, F. S. (2013). Peri-operative interventions producing better functional outcomes and enhanced recovery following total hip and knee arthroplasty: an evidence-based review. $B M C$ Medicine, 11(1), 37.

Kehlet, H., \& Dahl, J. B. (1993). The Value of "Multimodal" or "Balanced Analgesia" in Postoperative Pain Treatment. Anesthesia \& Analgesia,77(5), 1048-1056. doi:10.1213/00000539-199311000-00030

Koepke, E. J., Manning, E. L., Miller, T. E., Ganesh, A., Williams, D. G., \& Manning, M. W. (2018). The rising tide of opioid use and abuse: The role of the anesthesiologist. Perioperative Medicine, 7(16). doi:10.1186/s13741-018-0097-4

Ladha, K. S., Patorno, E., Huybrechts, K. F., Liu, J., Rathmell, J. P., \& Bateman, B. T. (2016). Variations in the use of perioperative multimodal analgesic therapy. Anesthesiology,124(4), 837-845. doi:10.1097/ALN.0000000000001034 
Lenz, E. R., \& Pugh, L. C. (2013). The Theory of Unpleasant Symptoms. In M. J. Smith \& P. R. Liehr (Eds.), Middle Range Theory for Nursing (3 ${ }^{\text {rd }}$ ed.). (165-195). New York, NY: Springer.

Lenz, E. R., Pugh, L. C., Milligan, R., A., Gift, A., Suppe, F. (1997). The middle range theory of unpleasant symptoms: An update. Advances in Nursing Science, 19(3), 14-27.

Manworren, R. C. (2015). Multimodal Pain Management and the Future of a Personalized Medicine Approach to Pain. AORN Journal,101(3), 307-318. doi:10.1016/j.aorn.2014.12.009

Mao, Y., Wu, L., \& Ding, W. (2016). The efficacy of preoperative administration of gabapentin/pregabalin in improving pain after total hip arthroplasty: A metaanalysis. BMC Musculoskeletal Disorders, 17(1). doi:10.1186/s12891-016-1231-4

Mistry, J. B., Chughtai, M., Elmallah, R. K., Le, S., Bonutti, P. M., Delanois, R. E., Mont, A. A. (2016). What influences how patients rate their hospital after total hip arthroplasty. Journal of Arthroplasty, (31)11, 2422-2425.

Nguyen, I., Haas, R. E., \& Pugh, L. (2017). The application of the Theory of Unpleasant Symptoms to the education and Practice of nurse anesthesists. Nursing \& Healthcare International Journal, 1(4). Retrieved from https://www.researchgate.net/profile/Richard_Haas2/publication/318856758_The _Application_of_the_Theory_of_Unpleasant_Symptoms_to_the_Education_and_ Practice_of_Nurse_Anesthetists/links/5989fdd80f7e9b9d44c9c039/TheApplication-of-the-Theory-of-Unpleasant-Symptoms-to-the-Education-andPractice-of-Nurse-Anesthetists.pdf 
NYU Langone Health's Department of Orthopedic Surgery. (2019). Press Release Research Points to ways to reduce opioid Use Following Common Orthopedic Surgeries. Accessed on June 1, 2019. Released March 12, 2019. Retrieved from https://nyulangone.org/press-release/research-points-to-ways-to-reduce-opioiduse-following-common-orthopedic-surgeries

Onda, A., Ogoshi, A., Itoh, M., Nakagawa, T., \& Kimura, M. (2016). Comparison of the effects of treatment with celecoxib, loxoprofen, and acetaminophen on postoperative acute pain after arthroscopic knee surgery: A randomized, parallelgroup trial. Journal of Orthopaedic Science, 21(2), 172-177.

doi:10.1016/j.jos.2015.11.005

O'Neal, J. B., Freiberg, A. A., Yelle, M. D., Jiang, Y., Zhang, C., Gu, Y., . . Wang, J. (2017). Intravenous vs Oral Acetaminophen as an Adjunct to Multimodal Analgesia After Total Knee Arthroplasty: A Prospective, Randomized, DoubleBlind Clinical Trial. The Journal of Arthroplasty,32(10), 3029-3033.

doi:10.1016/j.arth.2017.05.019

Pain. (2019). In The American Heritage Idioms Dictionary online. Retrieved from https://www.dictionary.com/browse/pain

Parvizi, J., \& Bloomfield, M. R. (2013). Multimodal Pain Management in Orthopedics: Implications for Joint Arthroplasty Surgery. Orthopedics,36(2), 7-14. doi:10.3928/01477447-20130122-51

Pitchon, D. N., Dayan, A. C., Schwenk, E. S., Baratta, J. L., \& Viscusi, E. R. (2018). Updates on multimodal analgesia for orthopedic surgery. Anesthesiology Clinics,36(3), 361-373. doi:10.1016/j.anclin.2018.05.001 
Politi, J. R., Davis, R. L., \& Matrka, A. K. (2017). Randomized Prospective Trial Comparing the Use of Intravenous versus Oral Acetaminophen in Total Joint Arthroplasty. The Journal of Arthroplasty,32(4), 1125-1127. doi:10.1016/j.arth.2016.10.018

Prabhakar, A., Mancuso, K. F., Owen, C. P., Lissauer, J., Merritt, C. K., Urman, R. D., \& Kaye, A. D. (2014). Perioperative analgesia outcomes and strategies. Best Practice \& Research: Clinical Anesthesiology,28(2), 105-115. http://doi.org/10.1016/j.bpa.2014.04.005

Rashidian, A., Eccles, M. P., \& Russell, I. (2008). Falling on stony ground? A qualitative study of implementation of clinical guidelines' prescribing recommendations in primary care. Health Policy,85(2), 148-161. doi:10.1016/j.healthpol.2007.07.011

Schmidt, P. C., Ruchelli, G. B., Mackey, S. C., \& Carroll, I. R. (2013). Perioperative gabapentioids: Choice of agent, dose, timing, and effects on chronic post surgical pain. Perioperative Gabapentinoids: Choice of Agent, Dose, Timing, and Effects on Post Surgical Pain,119(5), 1215-1221. doi:10.1097/ALN.0b013e3182a9a896 Schwenk, E. S., Goldberg, S. F., Patel, R. D., Zhou, J., Adams, D. R., Baratta, J. L., .. . Epstein, R. H. (2016). Adverse Drug Effects and Preoperative Medication Factors Related to Perioperative Low-Dose Ketamine Infusions. Regional Anesthesia and Pain Medicine,41(4), 482-487. doi:10.1097/aap.0000000000000416

Shah, A., Hayes, C. J., \& Martin, B. C. (2017). Characteristics of Initial Prescription Episodes and Likelihood of Long-Term Opioid Use - United States, 20062015. MMWR. Morbidity and Mortality Weekly Report,66(10), 265-269. doi:10.15585/mmwr.mm6610a1 
Singh, H., Banipal, R. P. S., \& Singh, B. (2016). Assessment of adequacy of pain management and analgesic use in patients with advanced cancer using the Brief Pain Inventory and Pain Management Index calculation. Journal of Global Oncology, 3(3), 235-241.

Singla, N. K., Parulan, C., Samson, R., Hutchinson, J., Bushnell, R., Beja, E. G., .. . Royal, M. A. (2012). Plasma and Cerebrospinal Fluid Pharmacokinetic Parameters After Single-Dose Administration of Intravenous, Oral, or Rectal Acetaminophen. Pain Practice, 12(7), 523-532. doi:10.1111/j.15332500.2012.00556.x

Soffin, E. M., \& YaDeau, J. T. (2016). Enhanced recovery after surgery for primary hip and knee arthroplasty: a review of the evidence. BJA: British Journal of Anesthesia, 117(suppl_3), iii62-iii72.

Stevenson, K. L., Neuwirth, A. L., \& Sheth, N. (2018). Perioperative pain management following total joint arthroplasty: A review and update to an institutional pain protocol. Journal of Clinical Orthopaedics and Trauma,9(1), 40-45. Retrieved from https://doi.org/10.1016/j.jcot.2017.09.014

Tornero, C. T., Fernandez Rodriguez, L. E., \& Valls, J. O. (2017). Multimodal analgesia and regional anesthesia. Spanish Journal of Anesthesiology and Resuscitation, 64(7), 401-405. doi: 10.1016/j.redar.2017.01.008

Trasolini, N. A., McKnight, B. M., \& Dorr, L. D. (2018). The opioid crisis and the orthopedic surgeon. The Journal of Arthroplasty, 33(11), 3379-3382.

U.S Department of Health \& Human Services. (2018). Draft report on pain management best practices: updates, gaps, inconsistencies, and recommendations. Retrieved 
June 6,2019, from https://www.hhs.gov/ash/advisorycommittees/pain/reports/2018-12-draft-report-on-updates-gaps-inconsistenciesrecommendations/index.htmlWashington, DC: Government Printing Office.

U.S. Department of Health \& Human Services. (2017). HHS Acting Secretary Declares Public Health Emergency to Address National Opioid Crisis. Retrieved August 20, 2019, from https://www.hhs.gov/about/news/2017/10/26/hhs-acting-secretarydeclares-public-health-emergency-address-national-opioid-crisis.html

Vadivelu, N., Mitra, S., Schermer, E., Kodumudi, V., Kaye, A. D., \& Urman, R. D. (2014). Preventative analgesia for postoperative pain control: a broader concept. Local an Regional Analgesia. Retrieved May 30, 2019, from http://doi.org/10.2147/LRA.S62160

Valencia, J. L., \& Leopo, A. K. (2017). Preemptive analgesia in orthopedic surgery: a literature review. Clinical Trials in Orthopedic Disorders, 2(4), 144.

Westrich, G. H., Birch, G. A., Muskat, A. R., Padgett, D. E., Goytizolo, E. A., Bostrom, M. P., ... YaDeau, J. T. (2019). Intravenous vs oral acetaminophen as a component of multimodal analgesia after total hip arthroplasty: A randomized, blinded trial. Journal of Arthroplasty,34(7), S215-S220. Retrieved from https://doi.org/10.1016/j.arth.2019.02.030

Whitmore, R., \& Whisenant, D. (2019, February 5). Pharmacy Times: Practical Information for Today's Pharmacists. Retrieved June 1, 2019, from https://www.pharmacytimes.com/contributor/marilyn-bulloch-pharmdbcps/2019/02/opioid-prescribing-limits-across-the-states 
Wolf, E. (2019, January 4). Southern New Hampshire University Library. Retrieved August 25, 2019, from https://libanswers.snhu.edu/friendly.php?slug=faq/215024

World Health Organization. (n.d.) Cancer. Retrieved June 1, 2019, from https://www.who.int/cancer/palliative/painladder/en/

Zusman, E. E. (2012). HCAHPS replaces Press Ganey survey as quality measure for patient hospital experience. Neurosurgery, 71(2), N21-N24. 
Appendix A: Data Collection Spreadsheet

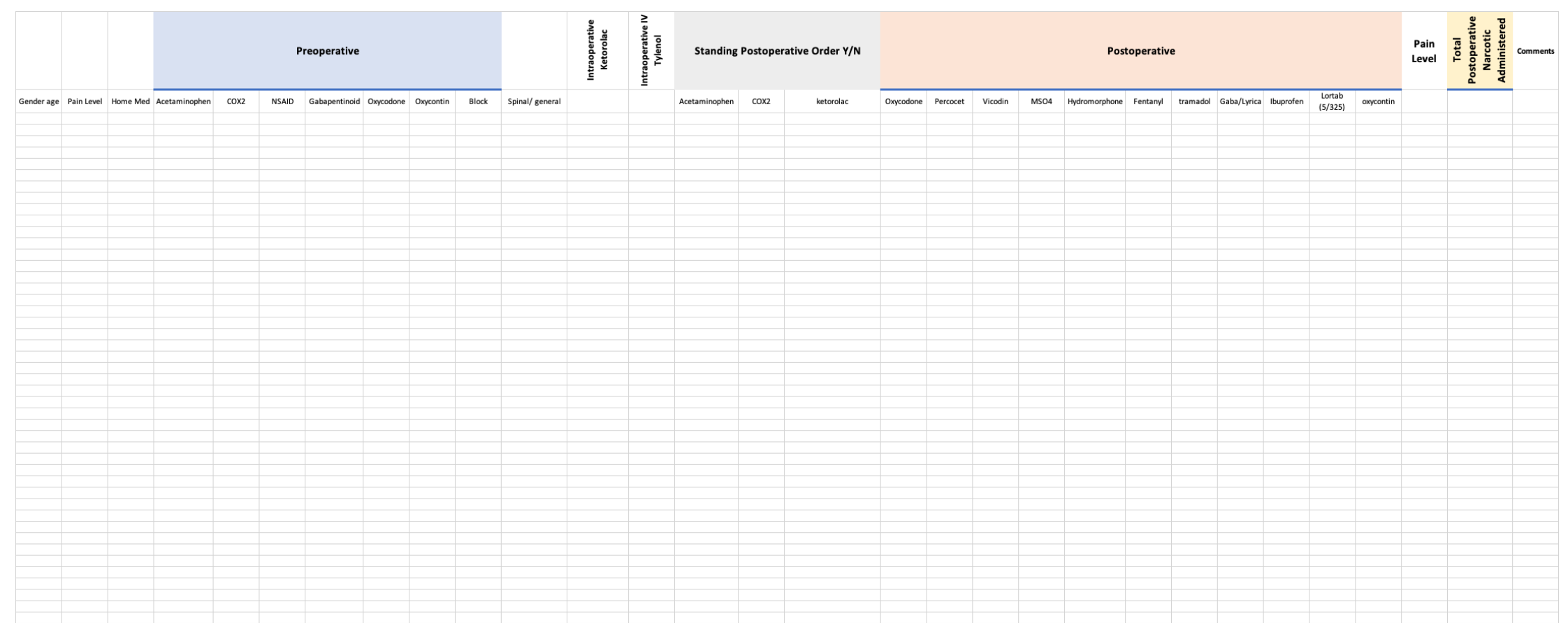




\section{Appendix B: IRB Approval Letter}

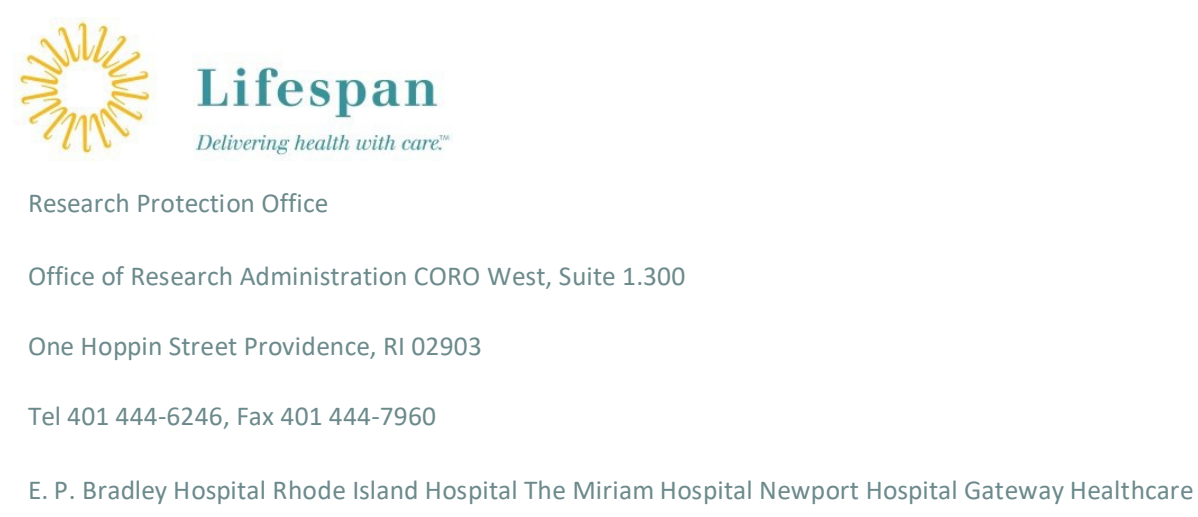

November 19, 2019

TO:

FROM:

SUBJECT:

PROJECT TITLE:

CMTT/PROJ:

ACTION:

EFFECTIVE DATE:

NEXT REPORT DUE:
John Fedo, PhD

Research Protection Office

IRB Determination of Exempt Human Subjects Research

[1472356-1 and 1472356-2] Preoperative Multimodal Analgesia in primary total hip arthroplasty

012919; 45 CFR 46.110(5)
EXEMPT

November 17, 2019

November 16, 2020

Thank you for your submission of New Project materials for this project. This package has received Exempt Review based on applicable federal regulations and institutional policy. The Lifespan - Rhode Island Hospital IRB 1 reviewed the New Project package as part of the above referenced project title in accordance with 45 CFR 46 and determined this project is EXEMPT from the requirements of 45 CFR 46.104 (d) for the following reasons:

1. The research is no more than minimal risk; and 
2. The only involvement of human subjects will be in one of more of the following categories of human subjects research: 45 CFR 46.104(d)(4).

3. The IRB conducted a limited IRB review to make the determination required by 45 CFR46.111(a)(7), when applicable.

This determination is based on the information provided in the submission. All research must be conducted in accordance with this determination.

If you have any questions, please contact Sara Spangenberger at (401) 444-6756 or sspangenberger@lifespan.org. Please include your project title and CMTT/PROJ or IRBNet ID in all correspondence with this committee.

\section{$\underline{\text { PI Responsibilities }}$}

It is the responsibility of the principal investigator to ensure that the study is conducted as approved by the IRB.

\section{Modifications and Amendments}

Exempt research does not require regulatory review. IRB review may be required if an investigator decides to modify an exempt human subjects research project in such a way that it would no longer qualify for exemption. Contact the Research Protection Office prior to implementation of any changes to the project to assure it continues to meet the criteria for EXEMPT status

$\underline{\text { Reporting of Events }}$

All NON-COMPLIANCE issues (including Protocol Deviations) or COMPLAINTS regarding this project must be reported promptly to this office.

\section{Protocol Expiration}

\section{A Progress Report is due by November 16, 2020 to confirm the research is still active.}

\section{STUDY CLOSURE AND RECORD RETENTION}

A Closure/Final Report is required at the conclusion of the human subjects research. Investigators are required to maintain research records for the longest period of time required by applicable Federal, State regulations and institutional policy (a minimum of 3-6 years).

\section{Lifespan Research Data Policy}

Any research data that includes Protected Health Information (PHI) or a Limited Data Set (LDS), as defined by HIPAA Regulations, may only be stored on: 
1. Lifespan managed storage platforms that comply with Lifespan policy "HSP-86.1 Data Backup and Storage Policy";

2. Lifespan managed computer workstations that comply with policy "HSP-90 Workstation Use Policy"; and

3. Mobile devices that comply with "HSP-102 Mobile Device Management Policy".

This includes data that originates from a Lifespan affiliated Covered Entity, personally identifiable information of Lifespan employees, or data originating from Lifespan or its affiliates that is classified as confidential.

For more information contact Lifespan IT department or Director, Research

Protection Office. IRB Compliance

The Lifespan - Rhode Island Hospital IRB 1 complies with HHS 45 CFR 46, FDA 21 CFR Parts 50 and 56

and other federal and state laws and regulations, as applicable, as well as ICH-GCP as they correspond to the FDA/DHHS regulations.

\section{Federalwide Assurance (FWA)}

Rhode Island Hospital (RIH): FWA00001230

The Miriam Hospital (TMH): FWA00003538

Emma Pendleton Bradley: FWA00001129

IRB00000482 Newport Hospital: FWA00003435

Gateway Healthcare: FWA00022347
OHRP IRB Registration

RIH IRB 1: IRB00000396

RIH IRB 2: IRB00004624

TMH IRB:

The following items are acknowledged in this submission:

- Application Form - Application Chart ReviewPart II revisions.pdf (UPDATED: 09/12/2019)

- Application Form - Appendix2-Full Waiver of Consent (UPDATED: 09/4/2019)

- CV/Resume - John M Fedo Resume 2018.doc (UPDATED: 09/1/2019)

- Data Collection - Template for data IRB info.docx (UPDATED: 09/12/2019)

- HIPAA Consent/Authorization - HIPAAPrepToResearch_V08142019.pdf (UPDATED: 09/9/2019)

- Lifespan - Research Application Part 1- Human Subject Studies - Lifespan - Research Application Part 1- Human Subject Studies (UPDATED: 09/10/2019)

- Protocol - Retrospective-Protocol-Template.doc (UPDATED: 09/12/2019)

- Training/Certification - HIPAA Security Certificate FEDO001.PDF (UPDATED: 11/12/2019)

- Training/Certification - CITI basic Bonnie Blaney 08.24.2019 (UPDATED: 08/25/2019)

- Other - IRB Modification letter.docx (UPDATED: 11/12/2019) 


\section{Appendix C: Protocol Data}

\section{Protocol Data}

Upon Lifespan Institutional Review Board approval, medical records will be reviewed to obtain a total of 100 qualifying cases. The review will begin with medical records dated August 31, 2019 to December 31, 2016. The chart reviewal process will begin after IRB approval date from Lifespan and the Rhode Island College.

Upon IRB approval, a request will be sent to Informational Services to obtain the following information from patient records who underwent total primary hip arthroplasty who are not deceased: age, gender, allergies, past medical history, home medications which includes last dose date and time, perioperative medications given, and inpatient medications in the first 24 hours. The medications obtained from inpatient record will include opioids, NSAIDs, acetaminophen, gabapentin, COX2 inhibitors (celecoxib), midazolam, diphenhydramine, and antiemetics (ondansetron). The opioid medications will include fentanyl, hydromorphone, morphine, tramadol, Percocet, Vicodin, oxycodone, and oxycontin. The NSAIDs will include ibuprofen, ketorolac, meloxicam, and diclofenac. The search will include whether spinal or general anesthesia was administered, and if a nerve blocks was performed. The patient's pain scores in the perioperative and inpatient areas will be in the perioperative and inpatient areas will be collected.

Data will be received in deidentified form from Informational Services and will be placed in the enclosed excel spreadsheet. All postoperative opioid dosages will be converted to Morphine equivalents using the Global $\mathrm{RxPh}$ conversion tool (https://globalrph.com/medcalcs/opioid-pain-management-converter-advanced/).

1. Approval from IRB from Lifespan and Rhode Island College

2. Submit request to IRB for information (age, gender, allergies, past medical history, home medications with last date and dose time, perioperative medications, inpatient medications in first 24 hours). Medications include opioids, NSAIDs, acetaminophen, gabapentin, COX2 inhibitors (celecoxib), midazolam, diphenhydramine, and antiemetics (ondansetron). The opioid medications will include fentanyl, hydromorphone, morphine, tramadol, Percocet, Vicodin, oxycodone, and oxycontin. The NSAIDs will include ibuprofen, ketorolac, meloxicam, and diclofenac. Type of anesthesia administered, and whether a block was administered.

3. Data will be placed into Excel Spreadsheet. (Submitted as data collection tool). 
4. Opioid dosage will be converted to morphine equivalent dosages from comparison. 